\title{
A post-translational modification signature defines changes in soluble tau correlating with oligomerization in early stage Alzheimer's disease brain
}

\author{
Ebru Ercan-Herbst ${ }^{1}$, Jens Ehrig ${ }^{2}$, David C. Schöndorf ${ }^{1}$, Annika Behrendt ${ }^{1}$, Bernd Klaus ${ }^{3}$, Borja Gomez Ramos ${ }^{1,4,5}$, \\ Nuria Prat Oriol ${ }^{1}$, Christian Weber ${ }^{1}$ and Dagmar E. Ehrnhoefer ${ }^{1 *}$ (D)
}

\begin{abstract}
Tau is a microtubule-binding protein that can receive various post-translational modifications (PTMs) including phosphorylation, methylation, acetylation, glycosylation, nitration, sumoylation and truncation. Hyperphosphorylation of tau is linked to its aggregation and the formation of neurofibrillary tangles (NFTs), which are a hallmark of Alzheimer's disease (AD). While more than 70 phosphorylation sites have been detected previously on NFT tau, studies of oligomeric and detergent-soluble tau in human brains during the early stages of AD are lacking. Here we apply a comprehensive electrochemiluminescence ELISA assay to analyze twenty-five different PTM sites as well as tau oligomerization in control and sporadic AD brain. The samples were classified as Braak stages 0-I, II or III-IV, corresponding to the progression of microscopically detectable tau pathology throughout different brain regions. We found that soluble tau multimers are strongly increased at Braak stages III-IV in all brain regions under investigation, including the temporal cortex, which does not contain NFTs or misfolded oligomers at this stage of pathology. We additionally identified five phosphorylation sites that are specifically and consistently increased across the entorhinal cortex, hippocampus and temporal cortex in the same donors. Three of these sites correlate with tau multimerization in all three brain regions, but do not overlap with the epitopes of phospho-sensitive antibodies commonly used for the immunohistochemical detection of NFTs. Our results thus suggest that soluble multimers are characterized by a small set of specific phosphorylation events that differ from those dominating in mature NFTs. These findings shed light on early PTM changes of tau during AD pathogenesis in human brains.
\end{abstract}

Keywords: Alzheimer's disease, Tau, Posttranslational modifications, Tau oligomerization

\section{Introduction}

Alzheimer's disease (AD) is the most common form of neurodegenerative diseases and is characterized pathologically by the presence of both neurofibrillary tangles (NFTs) and senile plaques [1-3]. While senile plaques are extracellular deposits of amyloid $\beta$-peptides [4], NFTs are formed intracellularly and consist of abnormally phosphorylated tau, a microtubule binding protein [5]. Mutations in the genes which affect the levels of amyloid $\beta$-peptide, such as APP (amyloid precursor

\footnotetext{
* Correspondence: ehrnhoefer@bio.mx

${ }^{1}$ BioMed X Innovation Center, Im Neuenheimer Feld 515, 69120 Heidelberg Germany

Full list of author information is available at the end of the article
}

protein), PSEN1 (Presenilin 1) and PSEN2 (Presenilin 2) cause familial AD (fAD) [6, 7]. On the other hand, sporadic AD (sAD), which accounts for more than $90 \%$ of all $\mathrm{AD}$ cases, is a multifactorial disease likely due to both genetic and environmental risk factors [8-10]. While sAD usually has a later onset compared to fAD, the disease progresses otherwise in a similar fashion [11, 12].

Both biomarker and neuropathological data show that tau pathology parallels cognitive dysfunction in $\mathrm{AD}$ more closely than amyloid $\beta$ pathology $[13,14]$. In particular, tau NFTs spread in a stereotypical manner throughout the brain, which has been used by Braak and colleagues as a method to differentiate disease stages [15]. In Braak stages I and II, which are very common in 
the elderly [13], NFTs are localized to the transentorhinal cortex. In Braak stages III and IV, the limbic regions such as hippocampus are additionally positive for NFTs. Finally, in Braak stages V and VI, neocortical involvement of NFTs is observed $[15,16]$.

While NFT formation is difficult to recapitulate in disease models and its exact cellular mechanisms remain to be further elucidated, it is well established that posttranslational modifications (PTMs) on tau protein have a role in this process $[17,18]$. Tau is heavily modified in both health and disease by several different PTMs such as phosphorylation, nitration, glycosylation, methylation, acetylation, sumolyation, ubiquitination and truncation $[19,20]$. Among all these different types of modifications, phosphorylation is studied most extensively [21]. Hyperphosphorylated tau molecules dissociate from microtubules and form detergent-soluble oligomeric structures, which later progress into detergent-insoluble aggregates [22]. The tau oligomer, an intermediate structure formed before the formation of NFTs, is thereby likely responsible for neuronal toxicity [23-28]. Even tau monomers were recently shown to be capable of adopting a conformation that promotes the seeding and spreading of pathology [2931]. To analyze different tau structures, conformationspecific antibodies have been developed, which are thought to react with the different folding states of the protein: Antibodies raised against oligomeric forms of tau such as T22, TOC1 and TOMA selectively label tau oligomers over monomers [24, 25, 32], whereas Alz50 and MC1 detect PHFs and NFTs $[33,34]$.

To date, many studies focusing on tau PTMs were carried out either under cell-free conditions, in cultured cell lines or in animal models. These studies provided valuable information on the enzymes modifying tau, such as kinases and phosphatases, and on the consequences of these modifications. For example, phosphorylation events at the sites T231, S235, S262, S293, S324, S356 decrease the affinity of tau to microtubules and result in destabilization of the neuronal cytoskeleton [35-37], while phosphorylation at C-terminal sites such as $\mathrm{S} 422$ promotes tau self-aggregation and can inhibit tau truncation at D421 [38, 39]. Studies using human brains are more limited, but several tau PTMs have been identified in postmortem samples using mass spectrometry and immunohistochemistry approaches, which we summarized previously (www.tauptm.org) [19]. However, most of these studies focused on PTMs present on NFTs, since detergent-soluble, oligomeric tau is more difficult either to discern by immunohistochemistry or to purify for mass spectrometry approaches.

ELISA-based techniques, on the other hand, are quantitative and allow for the detection of tau PTMs in whole tissue lysates [40]. We have previously established a panel of validated tau antibodies covering twenty-five
PTM sites [19], which we applied here to study tau PTMs in aged brains. We studied controls and sporadic $\mathrm{AD}$ samples ranging from Braak stages 0 to IV, and brain regions that are sequentially affected by tau pathology in AD: entorhinal cortex, hippocampus and temporal cortex. We furthermore developed an ELISA method to quantify non-monomeric tau species in detergent-soluble extracts and demonstrated that these species increase in all analyzed brain regions at Braak stages III-IV, in parallel with specific alterations in tau PTMs. Importantly, these PTMs were not changed at Braak stage II or in iPSC-derived neurons, where detergent-soluble tau multimers were also not detected. The pattern of altered tau PTMs was strikingly similar in all brain regions analyzed, which led us to define a tau PTM signature characteristic for early, disease-associated changes in $\mathrm{AD}$. These results thus advance our knowledge on tau pathology and have implications for future diagnostic and therapeutic approaches targeting tau.

\section{Methods \\ Human brain tissue lysate preparation}

Anonymized human post-mortem tissues (Table 1) were obtained from the London Neurodegenerative Diseases Brain Bank, a member of the Brains for Dementia Research Network. Lysates from human entorhinal cortices, hippocampi and temporal cortices were prepared in lysis buffer containing $150 \mathrm{mM} \mathrm{NaCl}, 20 \mathrm{mM}$ Tris $\mathrm{pH} 7.5,1$ mM EDTA, 1 mM EGTA, 1\% Triton-X100 and protease, phosphatase, demethylase (500 $\mu \mathrm{M}$ IOX1 (Active Motif), $2 \mu \mathrm{M}$ Daminozide (Active Motif), $10 \mu \mathrm{M}$ Paragyline Hydrochloride (Sigma)), deacetylase $(10 \mu \mathrm{M}$ Trichostatin A (Sigma), $5 \mathrm{mM}$ Nicotinamide (Sigma)), O-GlcNAcase $(1 \mu \mathrm{M}$ Thiamet-G (Sigma)) inhibitors. Lysis was performed with a dounce homogenizer. The homogenized lysates were spun down at $18000 \times \mathrm{g}$ at $4{ }^{\circ} \mathrm{C}$ for $30 \mathrm{~min}$. The supernatant was collected, and the protein concentration was measured by BCA assay according to manufacturer's instructions (BioRad).

\section{Electrochemiluminescence ELISA}

Meso Scale Discovery (MSD) Gold Streptavidin smallspot 96-well plates were blocked with 5\% (w/v) Blocker

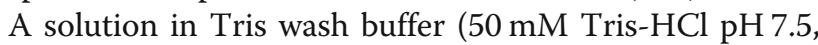
$150 \mathrm{mM} \mathrm{NaCl}$ and $0.02 \%$ Tween-20). Plates were sealed and allowed to block for $1 \mathrm{~h}$ at room temperature (RT) on a plate shaker. The plates were then washed three times with Tris wash buffer and coated with $25 \mu \mathrm{L}$ of biotinylated antibody diluted in 1\% Blocker A solution. The biotinylation of the antibodies was performed according to the manufacturer's instructions (EZ-Link Sulfo-NHS-Biotin, Cat No. 21217, Thermo Scientific). Before biotinylation, BSA was removed with the Melon Gel IgG Purification Kit (Cat. No 45212, Thermo Scientific), if 
Table 1 List of anonymized brain samples received from Brains for Dementia Research Network. EC: Entorhinal Cortex, Hip: Hippocampus, TC: Temporal Cortex

\begin{tabular}{|c|c|c|c|c|c|c|c|c|}
\hline ID & Sex & Age & Braak tangle stage & Thal phase & APOE genotype & Postmortem delay (h) & Tissues obtained & Brain bank \\
\hline Ctrl1 & M & 78 & 0 & 0 & $3 / 4$ & 56 & EC, Hip, TC & South West Dementia Brain Bank \\
\hline Ctrl2 & $\mathrm{F}$ & 86 & 1 & 1 & $3 / 3$ & 38.5 & EC, Hip, TC & South West Dementia Brain Bank \\
\hline Ctrl3 & $\mathrm{F}$ & 96 & 1 & 0 & $3 / 3$ & 49 & EC, Нiр, TC & South West Dementia Brain Bank \\
\hline Ctrl4 & M & 92 & 1 & 2 & $3 / 4$ & 56.5 & EC, Нiр, TC & South West Dementia Brain Bank \\
\hline Ctrl5 & $\mathrm{F}$ & 70 & 1 & 0 & $2 / 4$ & 55.5 & EC, Hip, TC & South West Dementia Brain Bank \\
\hline Ctrl6 & $\mathrm{F}$ & 69 & 0 & 1 & $3 / 4$ & 48 & EC, Нiр, TC & $\begin{array}{l}\text { London Neurodegenerative Diseases } \\
\text { Brain Bank }\end{array}$ \\
\hline Ctrl7 & M & 74 & 1 & 1 & $3 / 3$ & 24 & EC, Hip, TC & $\begin{array}{l}\text { London Neurodegenerative Diseases } \\
\text { Brain Bank }\end{array}$ \\
\hline Ctrl8 & M & 77 & 0 & 0 & $3 / 3$ & 11 & EC, Hip, TC & $\begin{array}{l}\text { London Neurodegenerative Diseases } \\
\text { Brain Bank }\end{array}$ \\
\hline AD1 & $\mathrm{F}$ & 83 & 2 & N/A & $3 / 4$ & 39 & EC, Нiр, TC & $\begin{array}{l}\text { London Neurodegenerative Diseases } \\
\text { Brain Bank }\end{array}$ \\
\hline AD2 & M & 80 & 2 & 1 & $3 / 3$ & 31 & EC, Hip, TC & $\begin{array}{l}\text { London Neurodegenerative Diseases } \\
\text { Brain Bank }\end{array}$ \\
\hline AD3 & $\mathrm{F}$ & 76 & 2 & N/A & $3 / 3$ & 22 & EC, Hip, TC & $\begin{array}{l}\text { London Neurodegenerative Diseases } \\
\text { Brain Bank }\end{array}$ \\
\hline AD4 & $\mathrm{F}$ & 86 & 2 & 1 & $3 / 3$ & 45 & EC, Hip, TC & $\begin{array}{l}\text { London Neurodegenerative Diseases } \\
\text { Brain Bank }\end{array}$ \\
\hline AD5 & M & 96 & 3 & 3 & $3 / 3$ & 25.5 & EC, Hip, TC & South West Dementia Brain Bank \\
\hline AD6 & $\mathrm{F}$ & 85 & 3 & 0 & $2 / 2$ & 13.5 & EC, Hip, TC & South West Dementia Brain Bank \\
\hline AD7 & $\mathrm{F}$ & 95 & 3 & 4 & $2 / 4$ & 65.5 & EC, Hip, TC & South West Dementia Brain Bank \\
\hline AD8 & $\mathrm{F}$ & 87 & 4 & 4 & $3 / 4$ & 71.5 & EC, Hip, TC & South West Dementia Brain Bank \\
\hline AD9 & M & 81 & 4 & 5 & $3 / 4$ & 38 & EC, Нiр, TC & South West Dementia Brain Bank \\
\hline
\end{tabular}

necessary. After incubating for $1 \mathrm{~h}$ at RT on a plate shaker, plates were washed three times with Tris wash buffer. For each sample $1 \mu \mathrm{g}$ of protein lysate (diluted in $50 \mu \mathrm{l} 1 \mathrm{xTBS}$ ) was incubated for $1 \mathrm{~h}$ at RT on a plate shaker. For analysis of denatured samples, samples were boiled in SDScontaining buffer $(62.5 \mathrm{mM}$ Tris- $\mathrm{HCl}$ pH 6.8, 10\% Glycerol, 2\% SDS) where the final amount of detergent did not exceed $0.02 \%$. Plates were washed three times with Tris wash buffer to get rid of unbound lysates and then incubated with $25 \mu \mathrm{l}$ of $0.5 \mu \mathrm{g} / \mathrm{ml}$ detection antibody (Tau12 labeled with MSD Sulfo-Tag-NHS-Ester, Cat. No: R31AA, Meso Scale Discovery) diluted in 1\% Blocker A solution for $1 \mathrm{~h}$ at RT on a plate shaker. The plates were then washed three times with Tris wash buffer. $150 \mu \mathrm{l}$ of $2 \mathrm{X}$ Read Buffer (Cat. No. R92TC, Meso Scale Discovery) were added 5 min before the signal was measured on a Meso Scale Discovery Quickplex platform.

\section{Antibodies}

The antibodies used in this study were characterized previously [19]. Information on the supplier and catalog numbers can be found in Table 2 .

\section{Statistical analysis of ELISA data}

Total tau intensity values were scaled within each sample type by dividing them by their geometric mean. The data was then normalized by the dividing the background-corrected signal intensity by the scaled total tau values. Subsequently, we used the generalized logarithm on the $\log 2$ scale to put our normalized values on the log2-scale [41]. We then removed all normalized values below 0 , which correspond to signal intensities below the background range.

We performed a differential analysis using the software package limma [42, 43]. For this, we created a design matrix that compares the fold change between the AD and control conditions within each of the tissues. In total, we performed 4 comparisons: EC-Braak-II vs. EC-Braak0-I, EC-Braak-III-IV vs. EC-Braak-0-I, Hip-Braak-III-IV vs. Hip-Braak-0-I, TC-Braak-III-IV vs. TC-Braak-0-I. Statistical significance was determined with an "omnibus" test (similar to an ANOVA procedure) to determine overall differences within the dataset and applied a FDR cutoff of $5 \%$ to obtain a list of candidate PTMs. Finally, individual comparisons within each tissue type were performed to determine the location of the change. 
Table 2 List of tau antibodies used in this study

\begin{tabular}{|c|c|c|c|}
\hline Name & Species & Company & Cat No. \\
\hline Tau12 & mouse & Biolegend & SIG-39416 \\
\hline Tau5 & mouse & Abcam & ab80579 \\
\hline Tau1 & mouse & Millipore & MAB3420 \\
\hline Dako & rabbit & Agilent Dako & A0024 \\
\hline BT2 & mouse & Thermo Fisher & MN1010 \\
\hline HT7 & mouse & Thermo Fisher & MN1000 \\
\hline T22 & rabbit & Sigma-Aldrich & ABN454-I \\
\hline nY18 & mouse & Biolegend & 829,701 \\
\hline$n Y 29$ & mouse & Millipore & MAB2244 \\
\hline ack280 & rabbit & Anaspec & AS-56077 \\
\hline meK311 & mouse & Biolegend & MMS-5102 \\
\hline C3-D421 & mouse & Millipore & $36-017$ \\
\hline pY18 & mouse & Novusbio & NBP2-42402 \\
\hline pT181 & rabbit & Thermo Fisher & 701,530 \\
\hline pS198 & rabbit & Abcam & ab79540 \\
\hline pS199 & rabbit & Thermo Fisher & 701,054 \\
\hline pS202 & rabbit & Anaspec & AS-28017 \\
\hline pS199/202 & rabbit & Thermo Fisher & $44-768 \mathrm{G}$ \\
\hline pT205 & rabbit & Abcam & ab181206 \\
\hline pT212 & rabbit & Abcam & ab51053 \\
\hline pS214 & rabbit & Thermo Fisher & PA5-35762 \\
\hline pT217 & rabbit & Thermo Fisher & $44-744$ \\
\hline pT231 & rabbit & Thermo Fisher & 701,056 \\
\hline pS235 & rabbit & Thermo Fisher & PIPA535761 \\
\hline pS238 & mouse & Abcam & ab128889 \\
\hline pS356 & rabbit & Abcam & ab51036 \\
\hline pS396 & rabbit & Thermo Fisher & $44-752 \mathrm{G}$ \\
\hline pS400 & rabbit & Anaspec & AS-54978 \\
\hline pS404 & rabbit & Thermo Fisher & 44-758G \\
\hline pS409 & rabbit & Abcam & ab4861 \\
\hline pS416 & rabbit & Abcam & ab119391 \\
\hline pS422 & rabbit & Abcam & ab79415 \\
\hline
\end{tabular}

\section{Recombinant tau protein purification}

Tau variants (full length protein and a fragment encoding amino acids 256-368) were cloned into the pET19b vector (Novagen) in between the NcoI and BamHI restriction sites. The pET19b-Tau plasmids were transformed into $E$. coli BL21(DE3) cells (Novagen). Cells were grown in LB supplemented with ampicillin at $37^{\circ} \mathrm{C}$ until OD600 reached 0.6-0.8. The expression of the tau proteins was induced by the addition of $1 \mathrm{mM}$ IPTG. The cells were then grown for an additional $3 \mathrm{~h}$ at $37^{\circ} \mathrm{C}$ and harvested by centrifugation. The cell pellet was resuspended in running buffer $(50 \mathrm{mM} \mathrm{Na}$-phosphate pH 7.0, $1 \mathrm{mM}$ EGTA and $1 \mathrm{mM}$ DTT) supplemented with cOmplete protease inhibitors (Roche), benzonase (Merck) and $10 \mu \mathrm{g} / \mathrm{ml}$ lysozyme (Sigma). The cells were lysed by 4 passages through an EmulsiFlex C3 (Avestin). After centrifugation and filtration, the cleared lysates were boiled for $20 \mathrm{~min}$ at $100^{\circ} \mathrm{C}$. After another centrifugation and filtration step the lysate was then loaded onto a combination of a HiTrap Q and a HiTrap SP column (GE Healthcare) pre-equilibrated with running buffer. After loading the sample, the HiTrap Q column was removed. The HiTrap SP column was washed with running buffer and eluted in a gradient to running buffer containing $300 \mathrm{mM} \mathrm{NaCl}$. The HiTrap SP elution fractions containing the tau proteins were concentrated using a $30 \mathrm{MWCO}$ or $3 \mathrm{MWCO}$ Amicon centrifugal filter unit (Merck) and loaded on a HiLoad 16/600 Superdex 75 pg size exclusion chromatography column (GE Healthcare) equilibrated with running buffer. After SDS-PAGE analysis, the elution fractions with the highest purity were pooled and quantified. The samples were aliquoted, flashfrozen in liquid nitrogen and stored at $-80^{\circ} \mathrm{C}$.

\section{Tau aggregation assay}

Aggregation of tau proteins was evaluated with a thioflavin $\mathrm{T}$ assay. $10 \mu \mathrm{M}$ of tau protein was mixed with 20 $\mathrm{mM}$ Tris pH 7.5 containing $100 \mathrm{mM} \mathrm{NaCl}, 1 \mathrm{mM}$ EDTA, $1 \mathrm{mM}$ DTT, $0.03 \mathrm{mg} / \mathrm{mL}$ heparin sodium salt and $30 \mu \mathrm{M}$ thioflavin T. Aggregation signal was measured every 30 min for a total duration of $40 \mathrm{~h}$ using a fluorescence plate reader (EX: $450 \mathrm{~nm}, \mathrm{EM}: 520 \mathrm{~nm}$ ) at $37^{\circ} \mathrm{C}$. In parallel, vials containing the same aggregation mix without thioflavin $\mathrm{T}$ were incubated at $37^{\circ} \mathrm{C}$ for indicated time points. Samples were then flash-frozen in liquid nitrogen before storage at $-80^{\circ} \mathrm{C}$. These samples were used for electrochemiluminescence analysis as follows: aggregation samples were thawed, sonicated for $30 \mathrm{~s}$ and diluted in $1 \mathrm{X}$ TBS. The samples were either boiled or not boiled in SDS-containing buffer $(62.5 \mathrm{mM}$ Tris- $\mathrm{HCl} \mathrm{pH} 6.8$, $10 \%$ Glycerol, 2\% SDS) for $10 \mathrm{~min}$ as indicated, the final amount of detergent in the sample did not exceed $0.02 \% .100 \mathrm{pg}$ of tau aggregation sample were added per well of an MSD Gold Streptavidin small-spot 96 well plate (Meso Scale Discovery). ELISA analysis was then performed as described above and previously [19].

\section{Immunoprecipitation of tau from EC lysates}

$100 \mu \mathrm{g}$ of entorhinal cortex lysates from Braak 0-I and Braak III-IV were used for immunoprecipitation with Tau12 antibody. Magnetic Protein G beads (Dynabeads, Thermo Fisher) were blocked with Pierce protein free TBS blocking buffer and the beads were incubated with $8 \mu \mathrm{g}$ of Tau12 antibody for $1 \mathrm{~h}$ at RT. The beads were washed with lysis buffer and incubated with $100 \mu \mathrm{g}$ of EC lysates overnight at RT. Next day, beads were washed with lysis buffer and bound protein was eluted with 
$100 \mu \mathrm{l}$ of $50 \mathrm{mM}$ Glycin $\mathrm{pH} 2.8$ and the $\mathrm{pH}$ was neutralized with Tris.

\begin{abstract}
Atomic force microscopy
Cluster sizes of tau oligomers were measured with atomic force microscopy (AFM). Braak 0-I and Braak III-IV entorhinal cortex Tau12-IP eluates were deposited on freshly cleaved mica sheets and incubated for 60 min in a closed chamber with $100 \%$ humidity to avoid evaporation. The samples were then washed by $5 \mathrm{x}$ buffer exchange with Tris buffer (50 mM Tris pH 7.5, $150 \mathrm{mM}$ $\mathrm{NaCl})$. Atomic force microscopy measurements were carried out with a NanoWizard4 AFM (JPK, Germany) operated in the "QI Advanced Imaging" mode using BLAC40TS cantilevers (Olympus, Japan). Cantilevers were calibrated using the automatic "contact-free" method of the JPK NanoWizard Control software. AFM images were acquired of $1 \times 1 \mu \mathrm{m}^{2}$ areas using a setpoint of 0.2 $\mathrm{nN}$, a z-length of $100 \mathrm{~nm}$ and a pixel time of $6 \mathrm{~ms}$. The "measured height" data were saved and further processed in the Gwyddion software (ver. 2.53) [44] as follows. Line levelling was done by subtracting firstorder polynomial fits from each scan line - for this, larger features of the image were masked. To remove noise, the "conservative denoise" and the "Gaussian" filter were applied to the images with their "size"-parameters set to 3 and 2 pixels, respectively. Cluster detection was carried out using the "Interactive $\mathrm{H}_{-}$Watershed" plugin from the "SCF MPI CBG" repository [45] of the software Fiji [46]. For each detected cluster the maximum height value was saved and statistics on all cluster heights were then obtained using the software MATLAB (MathWorks).
\end{abstract}

\section{Generation of hiPSC-derived neurons}

Donor information as well as cell line identifiers are summarized in Additional file 1: Table S1. iPSC lines Ctrl3, sAD3, fAD1, fAD2, fAD3 and fAD4 were obtained from StemBancc. Ctrl1, Ctrl2, sAD1 and sAD2 were generated using ReproRNA technology (Stem Cell Technologies) and characterized in detail elsewhere [47]. All iPSCs were differentiated into neurons following a cortical neuronal induction protocol [48] with minor modifications. iPSC colonies were dissociated using Versene (Invitrogen) and seeded at a density of 200,000 cells $/ \mathrm{cm}^{2}$ in mTesR (Stemcell Technologies) with $10 \mu \mathrm{M}$ Rock inhibitor (SelleckChem). The next day, the medium was switched to neural induction medium containing N2B27 Medium (50\% DMEM/F 12, 50\% Neurobasal, 1:200 N2, 1: 100 B27, 1\% PenStrep, 0.5 mM Non-essential amino acids, (all Invitrogen), $50 \mu \mathrm{M}$ ß-mercaptoethanol (Gibco), $2.5 \mu \mathrm{g} /$ $\mathrm{ml}$ insulin and $1 \mathrm{mM}$ sodium pyruvate (both Sigma)), $10 \mu \mathrm{M}$ SB431542 (Selleckchem) and $1 \mu \mathrm{M}$ Dorsomorphin (Tocris) and changed daily for 11 more days. On day 12, cells were split using Accutase (Invitrogen) to a density of
220,000 cells $/ \mathrm{cm}^{2}$ in N2B27 Medium containing $10 \mu \mathrm{M}$ Rock inhibitor and $20 \mathrm{ng} / \mathrm{ml}$ FGF2 (Peprotech). The medium was changed every third day without Rock inhibitor. On day 25, cells were split using Accutase to a density of $220,000 / \mathrm{cm}^{2}$ in final maturation medium containing N2B27 medium with $20 \mathrm{ng} / \mathrm{ml}$ BDNF, $10 \mathrm{ng} / \mathrm{ml}$ GDNF (both Peprotech), $1 \mathrm{mM}$ dibutyryl-cAMP (Sigma), $200 \mu \mathrm{M}$ ascorbic acid (Sigma) and $10 \mu \mathrm{M}$ Rock inhibitor (SelleckChem). The medium was changed every third day without Rock inhibitor until day 60.

\section{Microscopy}

iPSC derived neurons were seeded at day 40 in a density of 20,000 cells/well on a 96-well imaging microplate (Greiner) and fluorescence pictures were taken between day 50-60. For imaging, cells were washed once with PBS and fixed with 4\% PFA (Fisher Scientific) for $20 \mathrm{~min}$ at room temperature. Cells were permeabilized with $0.1 \%$ Triton X-100 (Sigma) in PBS for $10 \mathrm{~min}$ and blocked with 5\% BSA (Sigma) in PBS for $1 \mathrm{~h} \mathrm{RT}$ at room temperature. Primary antibodies were diluted in 5\% BSA in PBS and cells were incubated over night at $4{ }^{\circ} \mathrm{C}$. The next day, cells were washed $3 x$ with PBS and incubated with secondary antibodies for $1 \mathrm{~h}$ at room temperature in the dark. Afterwards, cells were washed again 3x with PBS and imaged with an Axio Observer D1 (Zeiss). Antibodies used for microscopy analysis of iPSC-derived neurons were: MAP 2 (Biolegend, 822,501), GABA (Sigma, A2052), NeuN (Sigma, MAB377), VGlut1 (Thermo Scientific, 48-2400), Tuj1 (Cell Signaling Technologies, 4466), Tbr1 (Abcam, ab183032).

\section{Results}

In this study, we used Triton-X100-soluble fractions from entorhinal cortices (EC), hippocampi (Hip) and temporal cortices (TC) from the same patients (Braak stages $0-\mathrm{I}$ and III-IV) to monitor differences in Tau PTMs between brain regions sequentially affected by tauopathy in AD. We additionally analyzed the EC, Hip and TC from donors classified as Braak II to investigate whether alterations in Tau PTMs would already be apparent at this stage. Donors from all groups were within the same age range (69-96 years, Table 1 ).

To detect changes in tau PTMs quantitatively, we used a previously established electrochemiluminescence ELISA assay, with a validated tau PTM antibody panel [19] (Table 2). Briefly, this consists of a sandwich ELISA approach, with PTM-specific tau capture antibodies and Tau12, a total tau antibody, for detection. We quantified a total of twenty-five PTM sites: nitrated tyrosine 18 (nY18) and nitrated tyrosine 29 (nY29), acetylated lysine 280 (acK280), methylated lysine 311 (meK311), caspase cleaved tau at aspartic acid 421 (C3-D421) and twenty phosphorylation sites, including one tyrosine (pY18), five 
threonine (pT181, pT205, pT212, pT217, pT231) and fourteen serine (pS198, pS199, pS199+202, pS202, pS214, pS235, pS238, pS356, pS396, pS400, pS404, pS409, pS416, pS422) modifications (Table 2). We then normalized the PTM signals to total tau determined with the Tau5-Tau12 ELISA pair. However, it is important to note that comparisons across different sites (antibodies) should be avoided due to potential differences in biotinylation efficiencies and binding affinities of the antibodies.

\section{Native Braak III-IV, but not Braak II brain extracts show extensive changes in all tau PTMs analyzed}

First we compared tau PTMs in the EC, Hip and TC from donors classified as Braak 0-I to those classified as Braak II [13]. While PTMs were present in all samples under investigation (Fig. 1 and Additional file 2: Figure S1), fold changes were small and not significant.

We therefore moved on to the comparison between Braak stages 0-I and III-IV, where we investigated tau PTMs in the EC, Hip and TC from the same donors. In this analysis, both EC and Hip tissues derived from Braak stages III-IV showed an increase in phosphorylation at most sites, with the exception of pT212, pT217, pS404 and pS409 (Fig. 2 a and b). In TC, this set of four was among the eight sites unaltered in Braak III-IV patients, while 12 sites were also significantly increased in this tissue (Fig. 2c). Among the non-phospho PTMs that are part of our panel [19], only cleavage at D421 was increased in all three brain regions, while nitration at Y18 showed a significant increase in the EC (Additional file 3: Figure S2). Although this reflects the expected severity of tauopathy in the different brain regions (EC > Hip > $\mathrm{TC}$ ), we were concerned that potential soluble tau oligomers may influence ELISA signals when an assembly containing more than one tau molecule is bound by each capture antibody. We therefore analyzed whether any multimeric tau structures were present in our samples.

Triton-X100-soluble brain fractions contain tau multimers and Braak III-IV ECs contain more of multimeric tau structures with heights differing between $10 \mathrm{~nm}-30 \mathrm{~nm}$ For the analysis of tau multimers in detergent-soluble brain extracts we established an ELISA that uses Tau12 both as the capture and the detection antibody. In monomeric tau, the Tau12 epitope will be blocked upon binding to the capture antibody and, as a consequence, the detection antibody will not be able to bind and no signal will be generated. In contrast, multimeric tau contains additional, free Tau12 epitopes on other tau molecules in the same structure and thus will give a signal. Such an approach of using monoclonal antibodies raised against tau has been previously applied to detect multimeric species $[40,49]$. During the oligomerization

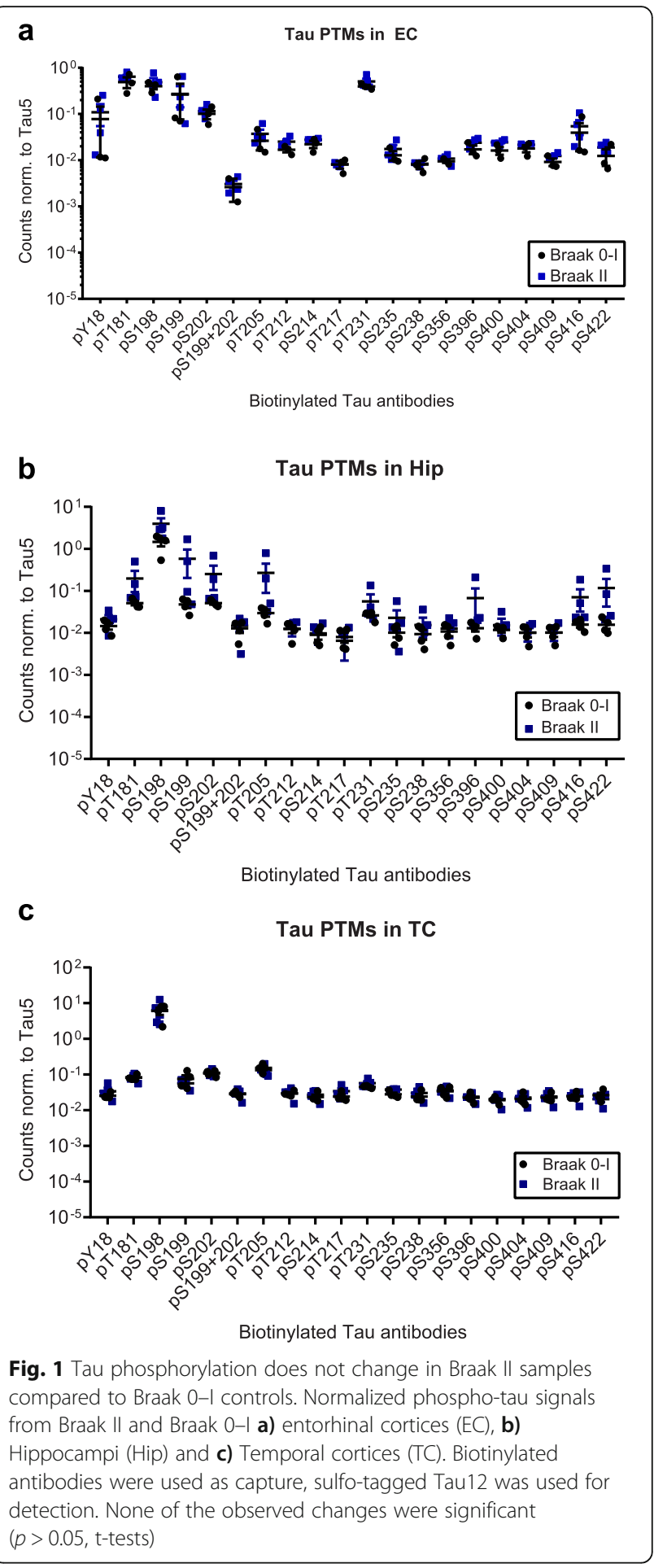

and aggregation process, tau furthermore undergoes a conformational shift which has been associated with toxicity and can be detected with conformation-specific antibodies such as T22 ([25, 50]).We therefore set up an additional ELISA method to detect oligomers containing misfolded tau using the conformation-specific antibody T22 as a capture and Tau12 for detection. 


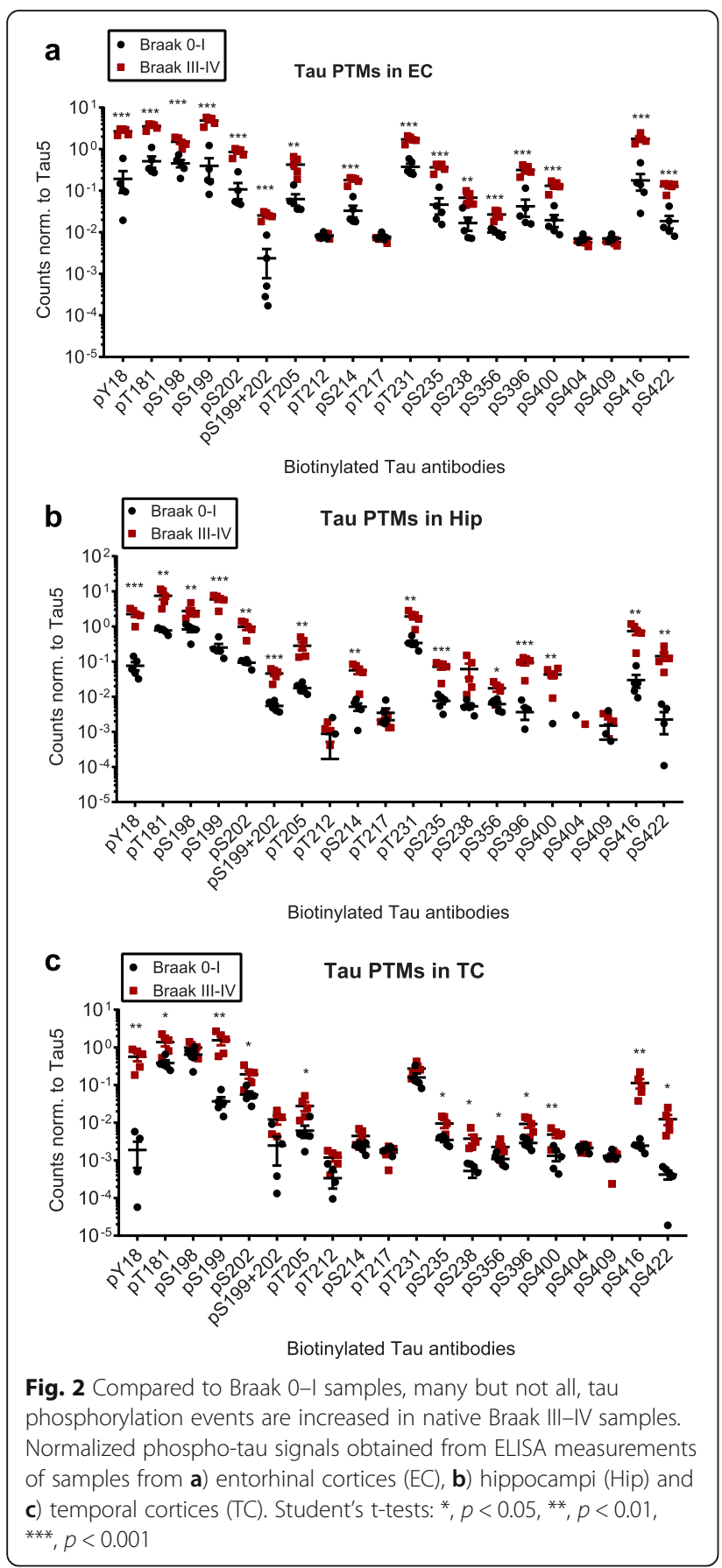

We first validated these methods using an in vitro aggregation assay with recombinant tau (2N4R). In parallel, we performed a Thioflavin $\mathrm{T}$ (ThT) binding assay to monitor the formation of beta sheet-containing structures as a readout for tau aggregation over time. Since full-length tau aggregation is a slow process in vitro, we added a pre-aggregated recombinant tau fragment encompassing the amino acids 256 to 368 as aggregation seeds [51]. As these seeds do not contain the Tau12 epitope, they should not interfere with the ELISA-based detection of full-length tau multimers. As expected, neither buffer nor seeds alone, nor full-length tau without seeds showed any increase in ThT signal over time (Fig. 3a). In contrast, the incubation of full-length tau with seeds led to an exponential increase in signal, slowing down after app. $8 \mathrm{~h}$ of incubation (Fig. 3a). Next, we performed an electrochemiluminescence ELISA with the Tau12-Tau12 pair to detect multimers. While we only observed a low baseline signal at the $0 \mathrm{~h}$ timepoint, the signal increased significantly for aggregated tau at $48 \mathrm{~h}$ (Fig. 3b). Interestingly, the signal of tau alone at $48 \mathrm{~h}$ also showed a significant increase, which was not detected by ThT assay. This suggests that compared to the ThT assay, the Tau12-Tau12 ELISA assay is more sensitive and detects additional non-monomeric tau species that may be either very small or do not contain $\beta$ sheet structures. Importantly, the signals from tau alone and tau with seeds at $48 \mathrm{~h}$ were completely abolished when the samples were boiled in SDS-containing buffer, confirming that the Tau12-Tau12 ELISA method can identify non-monomeric detergent-soluble tau species (Fig. 3b). In addition, the T22-Tau12 assay, which is expected to detect misfolded tau oligomers, showed a similar signal increase over time for both tau alone and tau with seeds, with the seeded aggregation reaction leading to the strongest signal, as expected (Fig. 3c). The boiling of samples with SDS-containing buffer abolished the signals, suggesting that the boiling process resolves oligomeric tau structures consistent with the Tau12-Tau12 assay. Moreover, dot blot analysis confirmed the time- and seedingdependent generation of T22-positive oligomers (Fig. 3d).

Using the same Tau12-Tau12 setup, we then determined the presence of tau multimers in EC, Hip and TC tissues from donors classified as Braak stages 0-I, II, or III-IV (Fig. 4a and b). While we did not detect any significant differences between Braak 0-I and Braak II (Fig. 4a), all brain regions from Braak III-IV resulted in a significantly increased ELISA signal, suggesting that tau multimers are present (Fig. 4b). On the other hand, the analysis of the Braak III-IV brain regions with the T22-Tau12 assay showed that only EC and Hip contain significantly increased misfolded tau oligomers, suggesting that the T22-Tau12 assay may only detect a subset of the multimeric tau species recognized by the Tau12Tau12 assay (Fig. 4c).

Tau oligomers associated with AD pathology have previously been reported to have diameters of $5-15 \mathrm{~nm}$ [50].To investigate the tau species in the EC of our Braak 0-I and Braak III-IV donors in more detail, we therefore immunoprecipitated tau with the Tau12 antibody and employed atomic force microscopy with quantitative image analysis. We found that for both Braak 0-I and III-IV, as well as for a negative control sample containing only Tau12 antibody without brain lysate, the atomically flat mica substrates are covered 


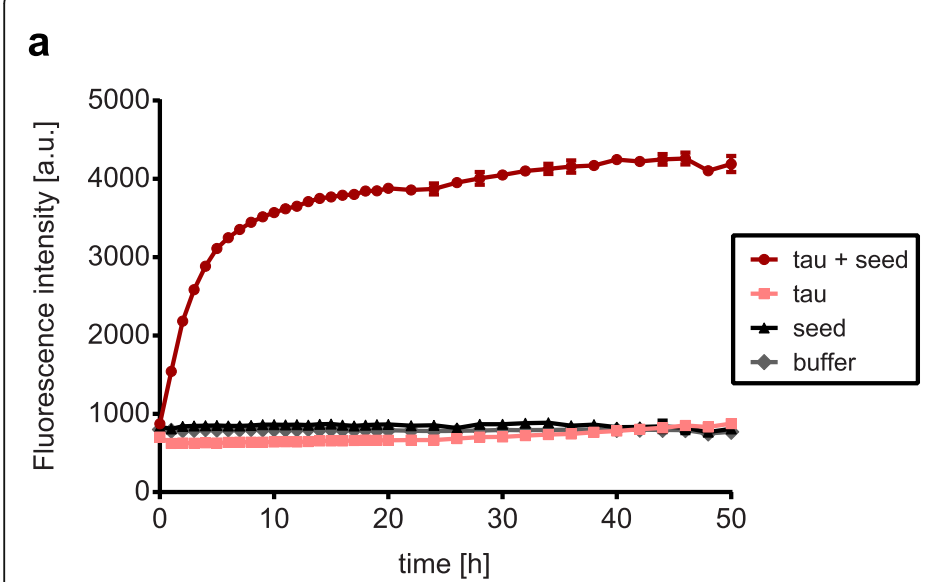

b

Tau12 - Tau12 assay

C T22 - Tau12 assay
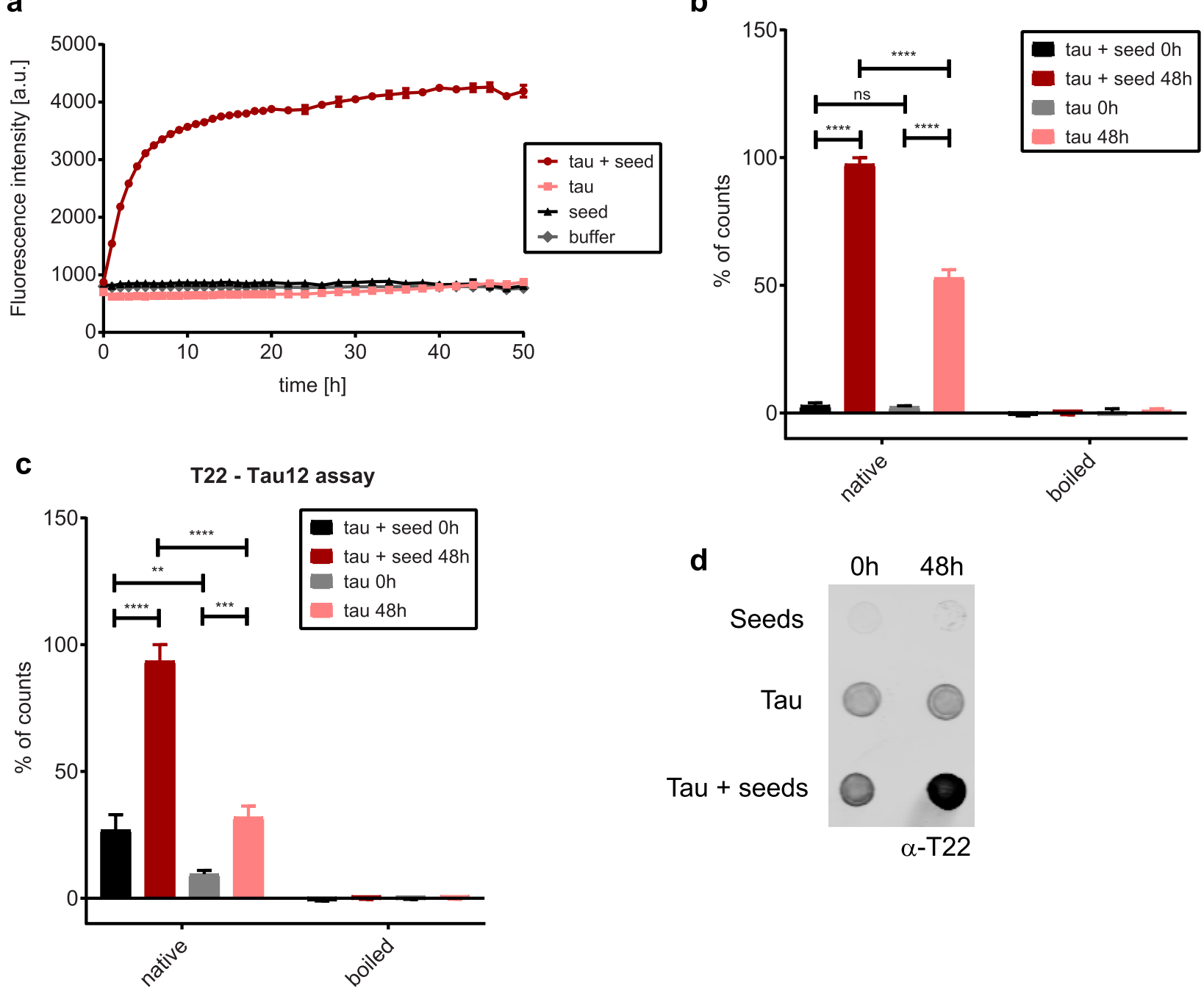

Fig. 3 Oligomerization of tau can be monitored with Tau12-Tau12 or T22-Tau12 ELISA. a) Fluorescence intensities of ThT assays showing aggregation of recombinant full-length tau over time. Seeds alone (tau aa256-368), buffer alone and full-length tau alone were used as controls. The signal for tau with seeds increases exponentially until app. $8 \mathrm{~h}$ of incubation $(n=3)$. Analysis of aggregates by b) Tau12-Tau12 ELISA assay and c) T22-Tau12 ELISA assay. Both methods yield a higher signal for tau with seeds after $48 \mathrm{~h}$ of incubation, which is abolished after boiling in SDS-containing buffer $(n=3)$. d) Dot blot analysis of native samples with T22 antibody: seeds alone, tau alone and tau with seeds at $0 \mathrm{~h}$ and $48 \mathrm{~h}$. Two-way Anova for $b$ and $c:{ }^{* *}, p<0.01,{ }^{* * *}, p<0.001,{ }^{* * *}, p<0.0001$

with an isotropic layer of molecules, leading to a topography with individual structures of up to $5 \mathrm{~nm}$ height. Clusters above $5 \mathrm{~nm}$ in height were only found in brain lysate samples. Here, the vast majority of clusters between 10 and $30 \mathrm{~nm}$ in height were detected in the Braak III-IV samples (Fig. 4d, e). This suggests that the significant increase of Tau12-Tau12 signal we observed in Braak III-IV EC may be due to these larger clusters.

We then asked whether the different amounts of multimeric structures detected by Tau12-Tau12 or T22Tau12 assays were due to different total levels of tau in the detergent-soluble fraction, and used six different total tau antibodies (HT7, BT2, Tau1, Tau5 and Dako-
Tau) raised against different domains of tau as capture antibodies and Tau12 as detection antibody (Fig. 5). While total tau levels in all Braak 0-I and Braak II samples did not show any differences (Fig. 5a-c), all three brain regions from Braak III-IV donors exhibited an increased signal only with HT7 as capture antibody but not with BT2, Tau1, Tau 5 and Dako-Tau antibodies (Fig. 5d-f).

Since these changes may be caused by tau multimers in the Braak III-IV samples, we next decided to assess whether boiling in SDS-containing buffer would resolve the difference to Braak 0-I tissue, similar to what we found for aggregates generated from recombinant tau 


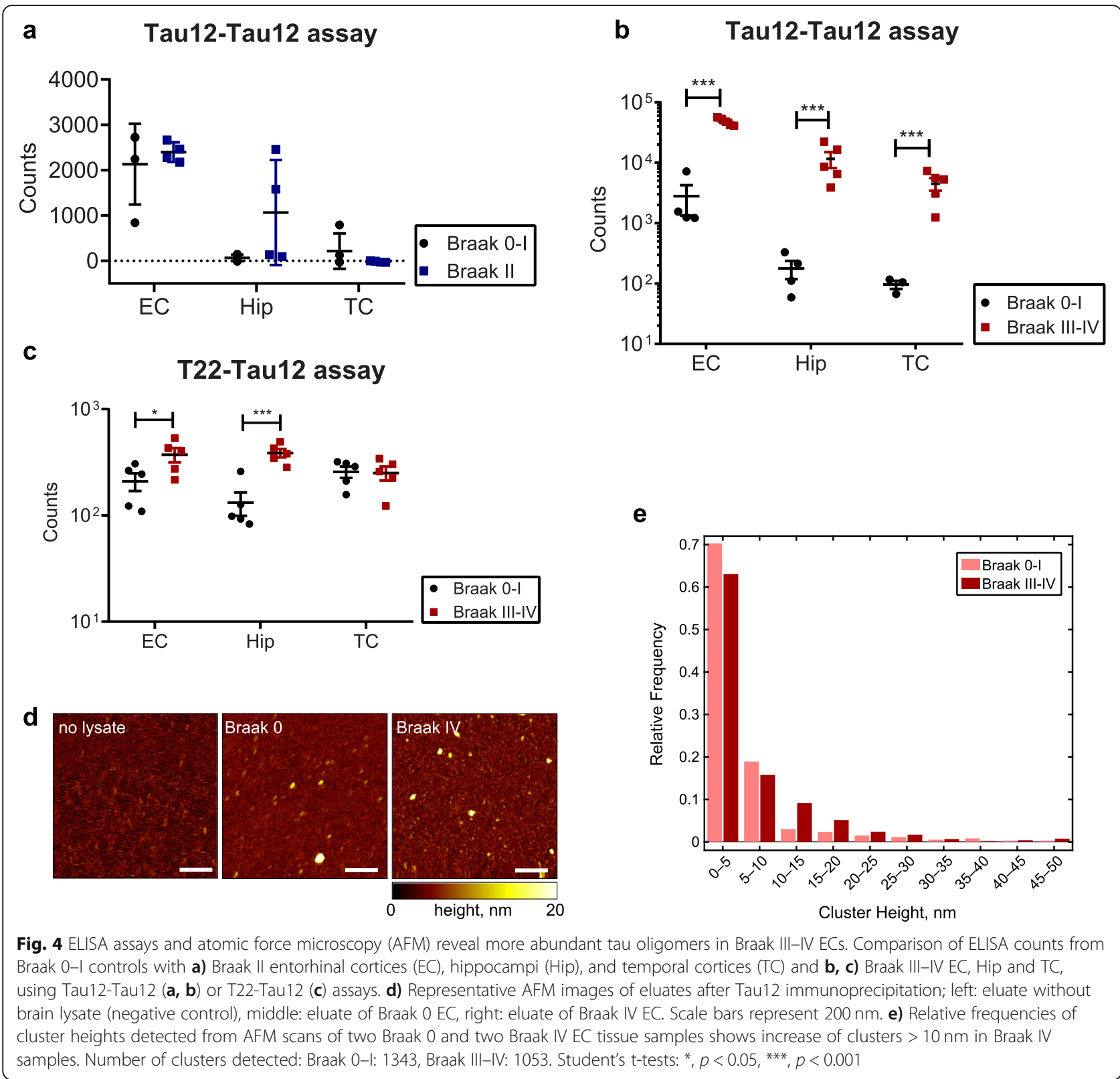

protein (Fig. 3b and c). Indeed, the denaturation treatment abolished the difference in Tau12-Tau12 ELISA signal between Braak 0-I and Braak III-IV samples for all three brain regions (Fig. 6a). Similarly, also the previously seen difference in HT7-Tau12 signal (Fig. 5d-f) was not observed when boiled Braak 0-I and Braak IIIIV EC, Hip and TC tissue samples were compared (Fig. 5b-d). Signals for all other total tau antibody combinations stayed similar between Braak stages, suggesting that the differences in Tau12-Tau12 and HT7-Tau12 signal in native samples were a result of tau multimerization, while the other antibody pairs were not as sensitive to aggregation state. Furthermore, these findings suggest that overall tau levels were not different between Braak stages in the Triton-soluble extracts.

Five consistently increased tau PTMs differentiate Braak stages 0 -I and III-IV

Since we had detected high levels of tau oligomers in all Braak III-IV samples, we next boiled the lysates with SDS-containing buffer and re-analyzed the PTM levels. Among the PTMs with previously observed increases (Fig. 2 and Additional file 3: Figure S2), this treatment dramatically reduced the differences between Braak stages (Fig. 7): In denatured samples, we found that the sites pS198, pS199, pT231, pS416 were significantly 


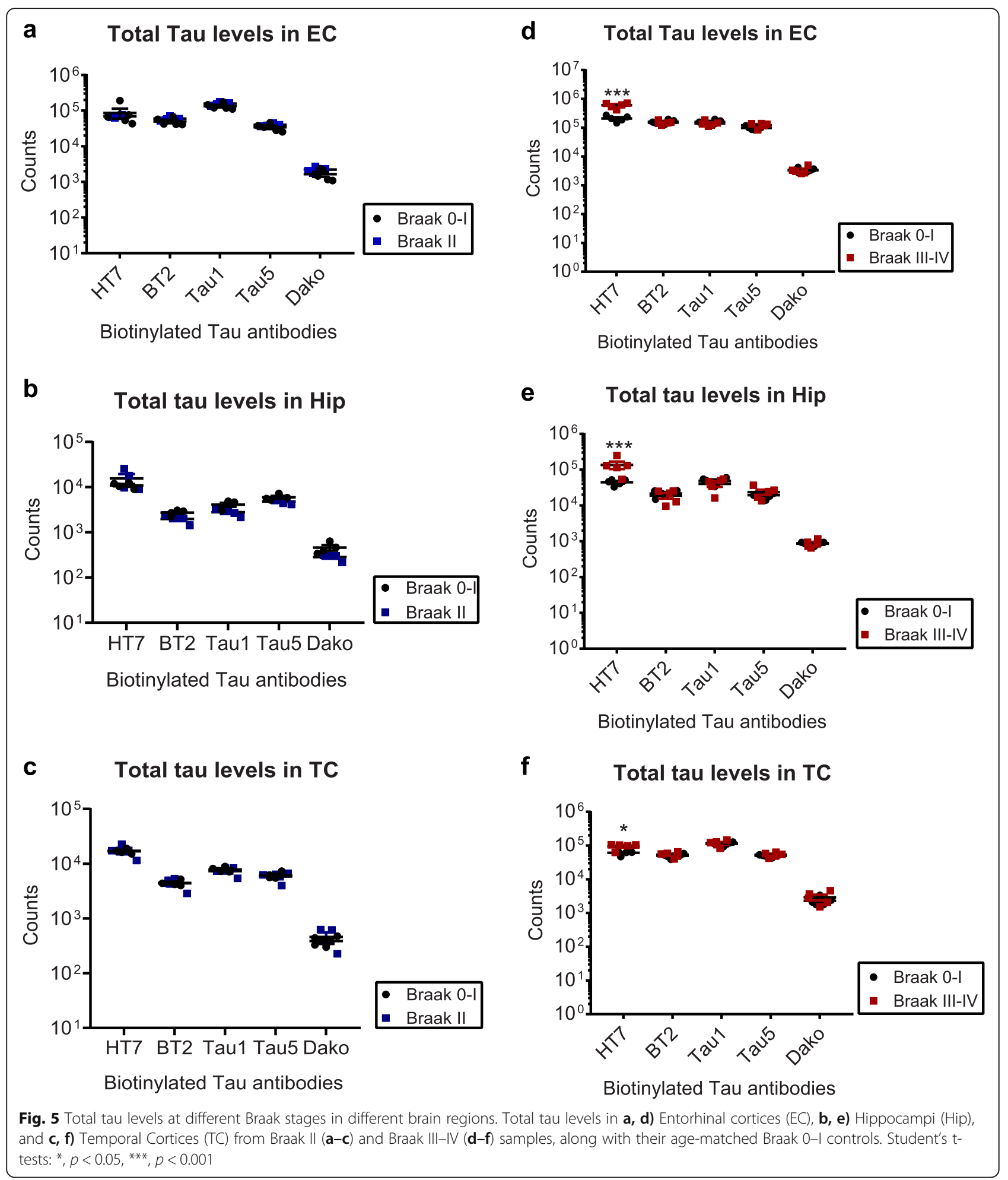

higher in the EC of Braak III-IV compared to Braak 0-I samples (Fig. 7a, b), in Hip tissue pY18, pS198, pS199, pT231, pS400, pS416 and pS422 were significantly increased at Braak stages III-IV (Fig. 7c, d), and in TC sites pS199 and pS416 were elevated in Braak III-IV compared to Braak 0-I (Fig. 7e, f).

Since there was a lot of overlap with regards to which PTMs were dysregulated in the different tissues, we next 


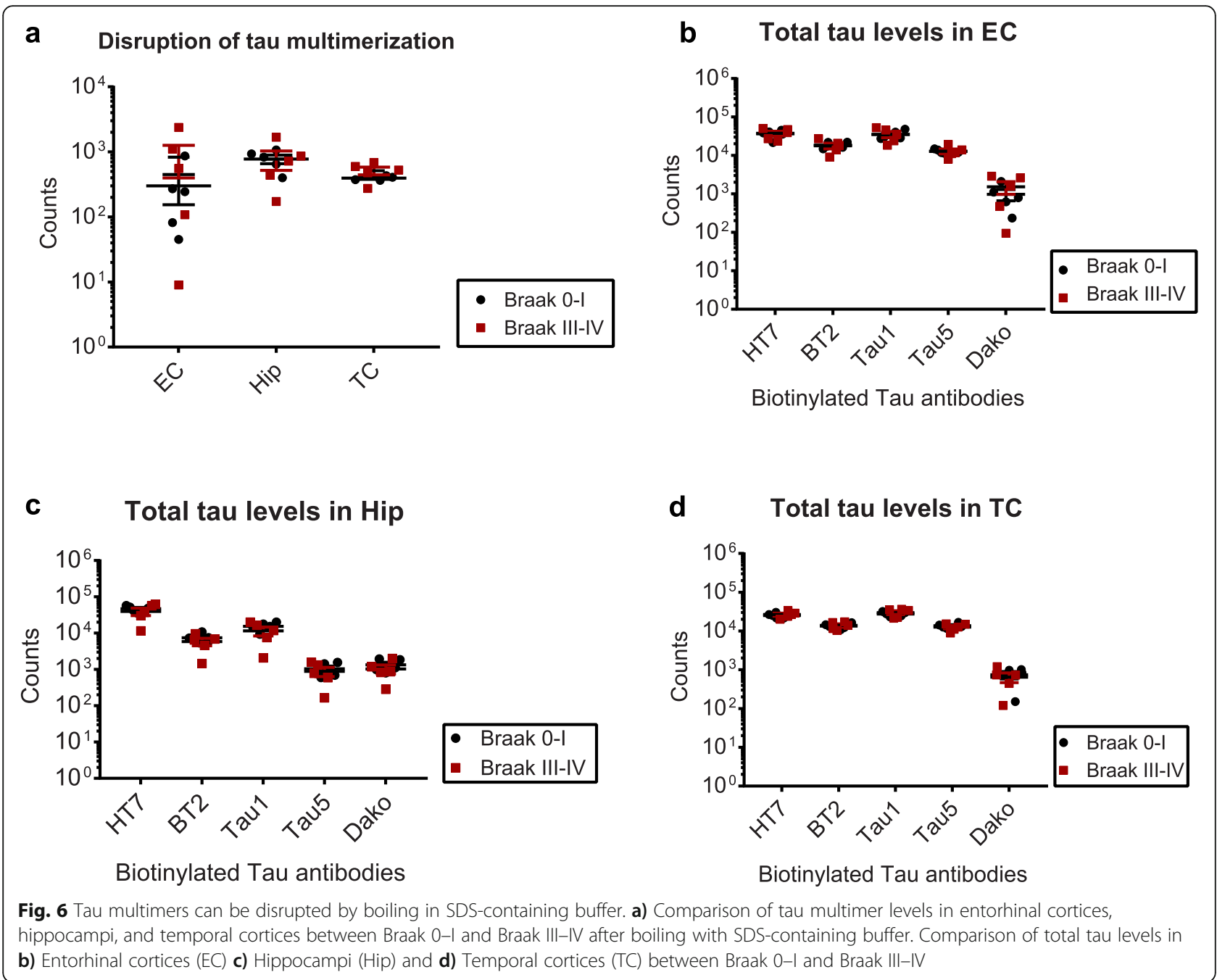

generated a linear model that takes changes in tau PTMs in four sample types into account: EC from Braak stage II, as well as EC, Hip and TC from Braak stages III-IV, in comparison to their respective Braak 0-I controls. This comparison revealed the sites pS198, pS199, pT231, pS416 and pS422 to be significantly (adj. $p$-value <0.01) increased over control in our cohort (Table 3).

\section{iPSC-derived neurons derived from sporadic and familial} $A D$ patients do not exhibit tau multimerization or aberrant tau PTMs

iPSC-derived neurons are an increasingly popular system to model neurodegenerative diseases in vitro, and lines generated from patient cells should in theory allow for disease modeling even in the absence of a familial mutation [52]. Nevertheless, these neuronal cultures represent an early developmental stage and there are conflicting reports as to whether $\mathrm{AD}$-related tau phenotypes can be observed [52-54]. We therefore decided to investigate whether Braak-stage dependent changes in tau
PTMs observed in brain tissue can be recapitulated in iPSC-derived neurons.

To this end, we generated cortical neurons from three control iPSC lines, three sporadic AD (sAD) and four familial AD (fAD) iPSC lines, each from a different donor fibroblast culture (Additional file 1: Table S1 and Additional file 4: Figure S3, [47]). From each line, we performed at least two independent differentiation rounds to assess variability. As our first readout, we checked whether tau multimers were present in SAD or fAD cells. Using the Tau12-Tau12 ELISA assay, we did not observe a consistent signal for any of the lines, and no change in signal was observed when lysates were boiled in SDS-containing buffer (Fig. 8a). This is in agreement with previous reports showing that the iPSCderived neurons do not contain any forms of multimeric or aggregated tau in the absence of additional triggers such as tau mutations, overexpression or seeding [55, 56]. Similarly, no significant differences were observed between control, sAD and fAD lines when comparing 


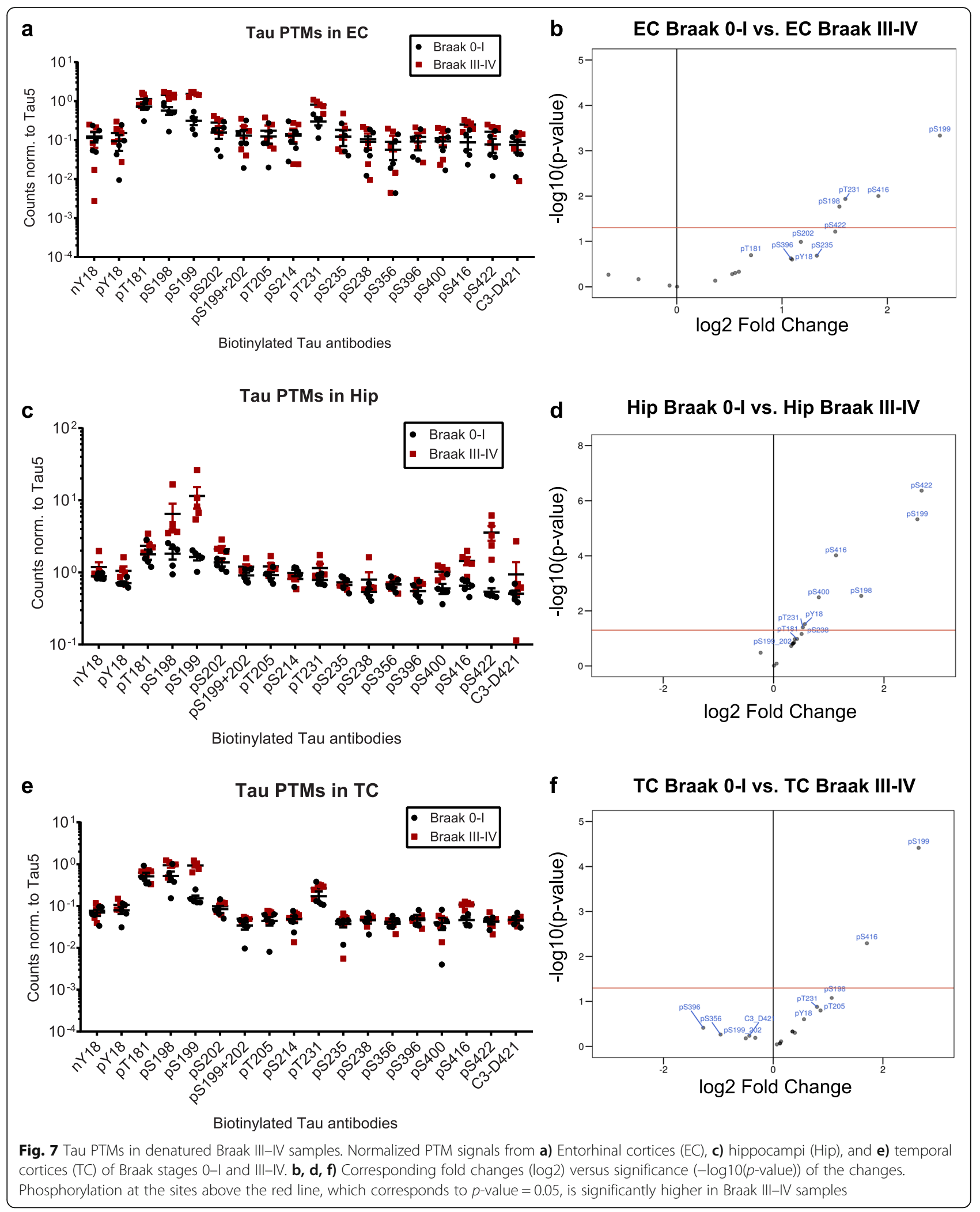


Table 3 Tau PTM events increased in at least one Braak III-IV tissue. Statistical significance was determined with Omnibus test

\begin{tabular}{lll}
\hline PTM events & $P$-value & adjusted $P$-value \\
\hline pS198 & 0.0007346 & 0.003306 \\
pS199 & 0.0000003316 & 0.000005968 \\
pT231 & 0.001081 & 0.003891 \\
pS416 & 0.0004662 & 0.002797 \\
pS422 & 0.00004812 & 0.0004331 \\
\hline
\end{tabular}

the levels of pS198, pS199, pT231 and pS416 - four sites that were significantly increased in brain tissues from Braak III-IV donors (Fig. 8b). Taken together, these findings suggest that the generation of iPSC-derived neurons with a cortical identity is not sufficient to consistently recapitulate changes in tau multimerization and PTM status that is observed in post-mortem patient tissues.

\section{Three PTMs correlate with tau multimerization}

Tau hyperphosphorylation increases its aggregation propensity in vitro $[57,58]$, and PHF-tau isolated from $\mathrm{AD}$ patient brains is heavily phosphorylated [59]. However, it remains unclear whether aggregation in vivo is driven by an increase in specific PTMs on soluble tau. We therefore tested whether the changes in tau PTMs observed in Braak III-IV brain tissues correlate with tau multimerization and the formation of misfolded oligomers. To this end, we performed a Spearman correlation analysis between the state of tau obtained by Tau12Tau12 and T22-Tau12 assay, and fold changes of all PTM sites for each individual denatured sample (Table 4). Multiple sites showed a strong $(r>0.5)$ and significant $(p<$ $0.05)$ correlation. The Tau12-Tau12 multimerization assay revealed that in the EC, phosphorylation events at sites
S198, S199, T231 and S416 correlated with multimerization. For Hip, pY18, pS198, pS199, pS202, pT205, pS238, pS396, pS400, pS416 and pS422 showed a positive correlation with tau multimerization, while a negative correlation was observed for pS214. Lastly, for TC, the sites pT181, pS198, pS199, pT231, pS416 correlated with tau multimerization. The T22-Tau12 oligomerization assay on the other hand did not reveal any correlation in EC, but in Hip the sites nY18, pY18, pS198, pS199, pT205, pS396, pS400, pS416 and pS422 showed a positive correlation (Table 4). Since no changes were detected with the T22-Tau12 ELISA in Braak III-IV TC (Fig. 4c), this tissue was not included in the correlation analysis for misfolded oligomers.

Among these phosphorylation events, pS198, pS199 and pS416 were consistently correlated with an increase in Tau12-Tau12 or T22-Tau12 ELISA signal in all brain regions analyzed (Table 4 and Fig. 9). Phosphorylation at these three sites also emerged as significantly increased in our analysis of PTM level differences (Table 3). Increases in pT231 levels, on the other hand, only correlated with multimerization in $\mathrm{EC}$ and $\mathrm{TC}$, while the increase in pS422 correlated with the Tau12-Tau12 and the T22-Tau12 signals only in Hip (Table 4). These findings suggest that three specific PTM sites are not only increased at early Braak stages, but their presence also strongly correlates with the formation of soluble tau multimers and misfolded oligomers, a marker of tau toxicity in AD.

\section{Discussion}

While tau dysfunction and toxicity has been linked to the formation of soluble oligomeric structures, these early intermediates are difficult to study in complex samples such as human brain. Therefore, much is known a

Multimerization of Tau in iPSC-derived neurons

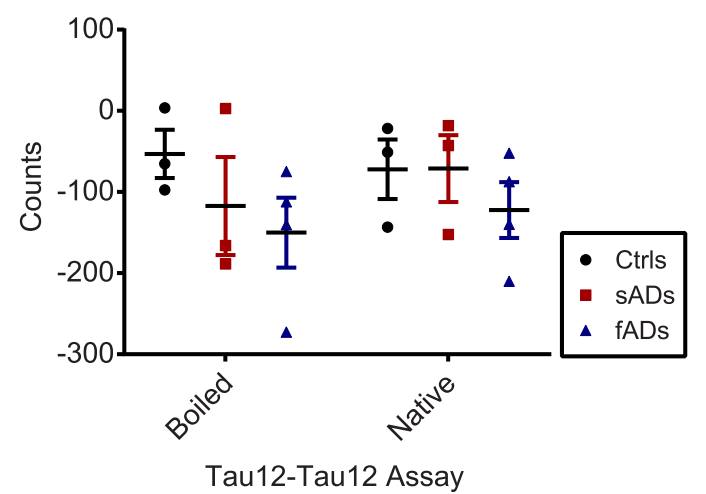

b

Tau PTM signature in iPSC-derived neurons

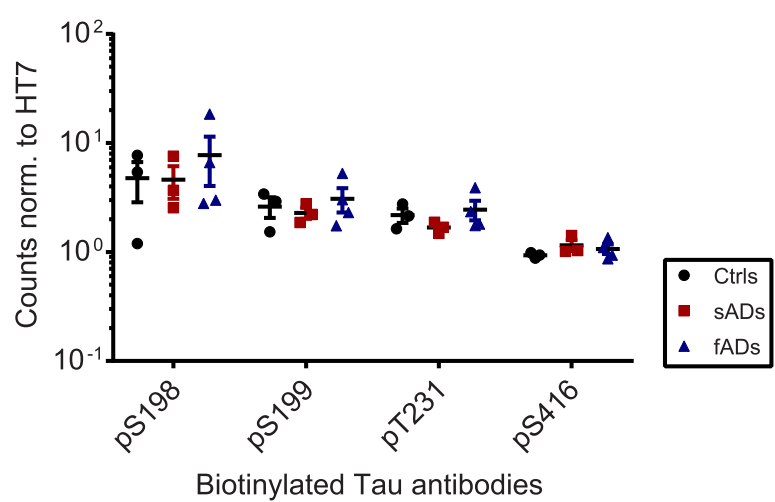

Fig. 8 iPSC-derived neurons do not recapitulate the tau PTM signature. a) Analysis of multimers by Tau12-Tau12 electrochemiluminescence assay with and without boiling of lysates from controls, familial AD ( $F A D$ ) and sporadic AD (SAD) neurons with SDS. b) Normalized PTM signals (pS198, pS199, pT231 and pS416). None of the observed changes were significant ( $p>0.05$, t-tests) 


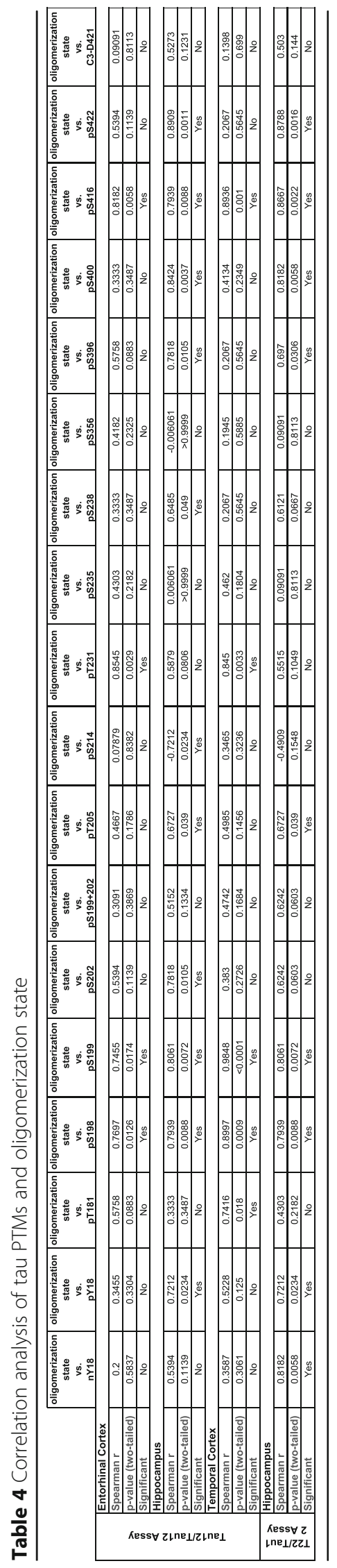


about PTMs and in particular tau phosphorylation on NFTs, but it is unclear whether the same sites are already differentially modified on soluble tau before aggregation. In this study we present a systematic analysis of PTM changes on soluble tau during early AD from human brain samples. While total tau levels are comparable between disease stages in these fractions, we do observe a strong shift particularly in tau phosphorylation during the progression from Braak stages 0-I to III-IV. Since many phospho-sites demonstrate an increased signal in native, but not in denatured Braak IIIIV samples, our data suggest that phospho-tau molecules form multimers together with non-modified tau, which thus provides additional binding sites for the Tau12 detection antibody. Interestingly, the sites showing a consistent increase in denatured samples are different from those that are traditionally used to stain NFTs and perform immunohistochemical Braak staging such as AT8 (pS202/pT205). However, despite the presence of antibodies against these phospho-sites in our panel, we did not observe an increase for their epitopes in the Triton-soluble fraction of Braak III-IV brains, although their signals did correlate with tau oligomerization in Hip tissue. This is in line with previous findings that the phospho-tau pattern differs during the development of NFTs, with specific phospho-sites being associated with pre-neurofibrillary tangles, intra- or extra-neuronal neurofibrillary tangles [60]. AT8 staining in particular is strongly associated with fibrillar aggregates [22], but has been observed in individuals as young as 20 years of age [61]. Braak and colleagues have therefore proposed that the occurrence of clinical AD symptoms may require synergistic effects between this age-dependent tauopathy and an additional insult [61]. Our results show a clear shift towards an increase of both tau multimerization and specific tau PTMs at Braak stages III-IV in the EC. Since AT8 staining in the EC is a defining feature already at Braak II, this suggests that tau pathology still increases in this brain region with disease progression.

Although most individuals at Braak III-IV are still clinically asymptomatic, we find biochemical manifestations of $\mathrm{AD}$ such as increased tau multimerization and phosphorylation even in the TC, which at this stage is largely AT8 negative. Importantly, we define a signature of three tau PTMs that is consistently increased and associated with multimerization throughout the EC, Hip and TC. Among the PTM events we identified, only pT231 has been previously linked to pre-tangle structures and was found increased at Braak stages corresponding to early disease (III-IV) [60, 62]. However, these studies were performed with a smaller antibody panel and by immunostaining, which is inherently less quantitative than ELISA. Furthermore, both pS199 and pT231 are increased in the cerebrospinal fluid (CSF) of
$\mathrm{AD}$ patients and are strongly increased in our samples, while pT181, a third commonly used CSF biomarker [63], did not differ between Braak stages in our study. pS416 and pS422, on the other hand, are likely too far at the tau C-terminus to be present on the truncated forms of tau detectable in CSF [64].

pS416 and pS422 were both previously described as being phosphorylated on synaptic tau in both human patients and mouse models [65-67]. pS422 in particular has been targeted by a passive immunization strategy in triple transgenic mice (TauPS2APP mice, [65]), and data from the same mouse model suggest that this phosphorylation event is promoted by the presence of amyloid plaques [66]. The fact that tau pS422 is most prominently changed in the Hip in our analysis therefore makes it tempting to speculate that this form of tau may actually be located synaptically in projections from excitatory pyramidal neurons in the EC, which are the most vulnerable neuron population at early stages of $\operatorname{AD}[68,69]$.

Misfolded tau oligomers are thought to be a major source of neuronal dysfunction in $\mathrm{AD}$, and we detected increased T22 signal in EC and Hip tissues, which also show the most alterations in PTMs at Braak stage III-IV. The increase in phosphorylation at the sites of our PTM signature may therefore alter the oligomerization and/or aggregation propensity of tau molecules, although such a connection still has to be formally demonstrated. Our correlation analysis between tau multimerization and PTM fold changes showed that pS198, pS199 and pS416 correlate with tau multimerization in all brain regions. A correlation with pT231 levels was only observed in EC and TC, while pS422 correlates with Tau12-Tau12 and T22 signals in Hip, where it is also most prominently increased. This argues against non-specific, general hyperphosphorylation of tau as a trigger of pathology and may thus be different from the physiological phosphorylation events occurring during development, anesthesia and hypothermia [20]. However, the factors responsible for the specific changes we observed remain unknown. Potential candidate enzymes include the kinases GSK3B, TTBK1, CAMK, PKA, CDK5 and the phosphatases PP2A and PP5 (www.tauptm.org) [19].

While further studies in human brain tissues are hampered by factors that influence enzymatic activities such as postmortem interval times [70], such studies are much easier to perform in model systems, and the use of iPSC-derived neurons for neurodegenerative disease research has revolutionized the field in the last years [71]. However, when we studied the tau PTM signature in iPSC-derived neurons from sporadic and familial AD patients, we found that the pattern we observed in human brains was not recapitulated, which might be due to their developmental immaturity and the absence of tau oligomerization in these cells. Developing cellular models 


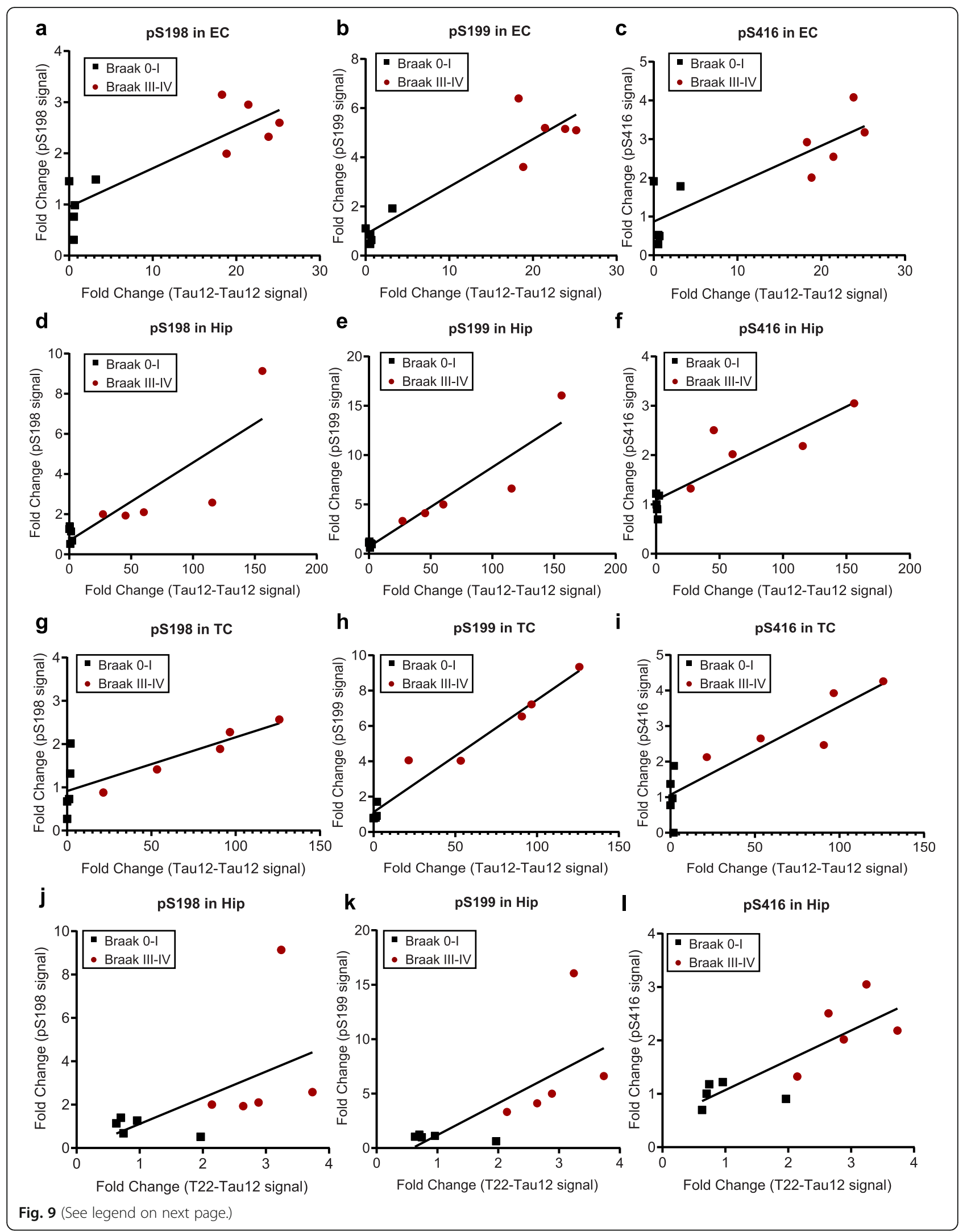


(See figure on previous page.)

Fig. 9 Correlation of tau oligomerization with pS198, pS199, and pS416 fold changes in all brain regions. Spearman correlation of the fold changes in Tau12-Tau12 signal with the fold changes (black squares: Braak 0-I / average (Braak 0-I); red circles: Braak III-IV / average (Braak 0-I)) of a) pS198, b) pS199 and c) pS416 in entorhinal cortex (EC), d) pS198, e) pS199 and f) pS416 in hippocampus (Hip), g) pS198, h) pS199 and i) pS416 in temporal cortex (TC) and Spearman correlation of the fold changes in T22-Tau12 signal with the fold changes (black squares: Braak 0-I / average (Braak 0-I), red circles: Braak III-IV / average (Braak 0-I)) of j) pS198, k) pS199 and I) pS416 in hippocampus (Hip). Results of the statistical analysis are summarized in Table 4

for AD and especially to study tau is challenging [56]. Despite many advantages, iPSC-derived neurons have the caveat that they express only one out of six isoforms of tau [53], and reprogramming results in the loss of aging factors, which may affect disease pathology [54, 72]. Using isogenic controls can be helpful to discern subtle disease phenotypes, however this is not an option for sporadic diseases without a single genetic cause [52].

For tau phosphorylation, previous studies have yielded variable results with some, but not all sporadic AD lines showing an increase [73, 74]. For familial AD, tau phenotypes have been reported for lines containing APP, but not presenilin mutations $[75,76]$. As three out of our four familial AD lines had PS1 mutations, this may be a reason for the lack of tau phenotypes in our cultures. Furthermore, a new study has also revealed that inter-laboratory variability is the largest source of failed reproducibility of experiments performed by iPSC-derived neurons [77].

With the advent of more complex culture systems such as 3D and co-culture models, it remains to be seen if iPSC technology can yield more robust phenotypes for sporadic and age-dependent disease in the future.

\section{Supplementary information}

Supplementary information accompanies this paper at https://doi.org/10. 1186/s40478-019-0823-2

\section{Additional file 1: Table S1. List of iPSC-derived neurons used in the study.}

Additional file 2: Figure S1. Non-phospho tau PTMs do not change in Braak II. Normalized tau PTM signals (nY18, nY29, Ack280, meK311, C3D421) in Braak II a) Entorhinal cortices (EC) b) Hippocampi (Hip) and c) Temporal cortices (TC) compared to Braak $0-\mid$ controls. None of the observed changes were significant ( $p>0.05$, t-tests).

Additional file 3: Figure S2. Specific increase in tau proteolysis at D421 and nitration at Y18 in native Braak III-IV compared to Braak 0-I samples. a, b, c) Normalized tau PTM signals (nY18, nY29, Ack280, meK311, C3D421) from native Braak III-IV and Braak 0-I entorhinal cortices, hippocampi and temporal cortices. Student's t-tests: ${ }^{*}, p<0.05,{ }^{* *}, p<0.01,{ }^{* * *}$, $p<0.001$ (t-tests).

Additional file 4: Figure S3. Differentiation of iPSCs from control, $\mathrm{AAD}$ and SAD donors to cortical neurons. Representative microscopy images of iPSC-derived neurons stained for neuronal markers a) MAP 2 (red), GABA (green) b) vGlut (red), NeuN (green) c) MAP 2 (green), Tau12 (red) and d) Tuj1 (green) and Tbr1 (red) and DAPI for nuclei (blue). Scale bars represent $50 \mu \mathrm{m}$ for all images.

\section{Acknowledgements}

The authors thank Dr. Martin Fuhrmann and Dr. Laura Gasparini for fruitful discussions and advice, Dr. Theron Johnson for access to the Meso Scale Discovery Quickplex platform and Dr. Kim Remans and Dr. Jacob Scheurich at the EMBL protein purification core facility for the expression and purification of recombinant tau proteins. Human post-mortem tissue was obtained from the London Neurodegenerative Diseases Brain Bank and the University of Bristol brain banks, members of the Brains for Dementia Research Network.

\section{Authors' contributions}

EEH performed the majority of the experiments, BGR and NPO helped with ELISA experiments, JE performed AFM experiments, DCS and CW provided iPSC-derived neurons, AB performed in vitro aggregation assays, BK performed statistical analyses. EEH coordinated author contributions and wrote the first draft of the manuscript. DEE designed the study, supervised the work and finalized the manuscript. All authors approved the final version.

\section{Funding}

The study was funded by a contract research agreement between AbbVie $\mathrm{GmbH} \& \mathrm{Co} \mathrm{KG}$ and BioMed X GmbH.

\section{Availability of data and materials}

The datasets during and/or analysed during the current study available from the corresponding author on reasonable request.

\section{Ethics approval and consent to participate}

Human brain samples were collected with informed consent by the London Neurodegenerative Diseases Brain Bank and the Southwest Dementia Brain Bank and were provided in a strictly anonymized fashion. hiPSCs were derived from fibroblasts that are part of the NIA Aging Cell Repository at the Coriell Institute for Medical Research [47], or obtained from StemBANCC. Informed consent was obtained by Coriell or StemBANCC, respectively, and the fibroblasts and hiPSCs were provided in a strictly anonymized fashion.

\section{Consent for publication}

Not applicable

\section{Competing interests}

The authors declare that they have no competing interests.

\section{Author details}

${ }^{1}$ BioMed X Innovation Center, Im Neuenheimer Feld 515, 69120 Heidelberg, Germany. ${ }^{2} \mathrm{~B}$ CUBE - Center for Molecular Bioengineering, Technische Universitaet Dresden, 01307 Dresden, Germany. ${ }^{3}$ Centre for Statistical Data Analysis, European Molecular Biology Laboratory (EMBL), 69117 Heidelberg, Germany. ${ }^{4}$ Present address: Life Sciences Research Unit, University of Luxembourg, L-4367 Belvaux, Luxembourg. ${ }^{5}$ Present address: Luxembourg Centre for Systems Biomedicine, University of Luxembourg, L-4362 Esch-sur-Alzette, Luxembourg.

Received: 23 September 2019 Accepted: 29 September 2019 Published online: 03 December 2019

References

1. Alzheimer A (1907) Über eine eigenartige Erkrankung der Hirnrinde. Allgemeine Zeitschrift fur Psychiatrie und Psychisch-gerichtliche Medizin 64: $146-148$

2. Ince PG, MCArthur FK, Bjertness E, Torvik A, Candy JM, Edwardson JA (1995) Neuropathological diagnoses in elderly patients in Oslo: Alzheimer's disease, Lewy body disease, vascular lesions. Dementia 6: 162-168

3. Bowler JV, Munoz DG, Merskey H, Hachinski V (1998) Fallacies in the pathological confirmation of the diagnosis of Alzheimer's disease. J Neurol Neurosurg Psychiatry 64: 18-24 
4. Dickson DW (1997) Neuropathological diagnosis of Alzheimer's disease: a perspective from longitudinal clinicopathological studies. Neurobiol Aging 18: S21-26

5. Braak H, Braak E (1998) Evolution of neuronal changes in the course of Alzheimer's disease. J Neural Transm Suppl 53: 127-140

6. Sorbi S, Forleo P, Tedde A, Cellini E, Ciantelli M, Bagnoli S, Nacmias B (2001) Genetic risk factors in familial Alzheimer's disease. Mech Ageing Dev 122: 1951-1960

7. Tanzi RE, Bertram L (2005) Twenty years of the Alzheimer's disease amyloid hypothesis: a genetic perspective. Cell 120: 545-555 https://doi.org/10.1016/ j.cell.2005.02.008

8. Alonso Vilatela ME, Lopez-Lopez M, Yescas-Gomez P (2012) Genetics of Alzheimer's disease. Arch Med Res 43: 622-631 https://doi.org/10.1016/j. arcmed.2012.10.017

9. International Genomics of Alzheimer's Disease C (2015) Convergent genetic and expression data implicate immunity in Alzheimer's disease. Alzheimers Dement 11: 658-671 https://doi.org/10.1016/j.jalz.2014.05.1757

10. Lahiri DK, Maloney B, Basha MR, Ge YW, Zawia NH (2007) How and when environmental agents and dietary factors affect the course of Alzheimer's disease: the "LEARn" model (latent early-life associated regulation) may explain the triggering of AD. Curr Alzheimer Res 4: 219-228

11. Atwood CS, Bowen RL (2015) A Unified Hypothesis of Early- and Late-Onset Alzheimer's Disease Pathogenesis. J Alzheimers Dis 47: 33-47 https://doi. org/10.3233/JAD-143210

12. Hunter S, Arendt T, Brayne C (2013) The senescence hypothesis of disease progression in Alzheimer disease: an integrated matrix of disease pathways for FAD and SAD. Mol Neurobiol 48: 556-570. https://doi.org/10.1007/ s12035-013-8445-3

13. Braak H, Del Tredici K (2015) The preclinical phase of the pathological process underlying sporadic Alzheimer's disease. Brain 138: 2814-2833. https://doi.org/10.1093/brain/awv236

14. Jack CR, Jr., Knopman DS, Jagust WJ, Petersen RC, Weiner MW, Aisen PS, Shaw LM, Vemuri P, Wiste HJ, Weigand SDet al (2013) Tracking pathophysiological processes in Alzheimer's disease: an updated hypothetical model of dynamic biomarkers. Lancet Neurol 12: 207-216 https://doi.org/10.1016/S1474-4422(12)70291-0

15. Braak H, Braak E (1991) Neuropathological stageing of Alzheimer-related changes. Acta Neuropathol 82: 239-259

16. Braak H, Alafuzoff I, Arzberger T, Kretzschmar H, Del Tredici K (2006) Staging of Alzheimer disease-associated neurofibrillary pathology using paraffin sections and immunocytochemistry. Acta Neuropathol 112: 389-404 https:// doi.org/10.1007/s00401-006-0127-z

17. Braak H, Braak E (1995) Staging of Alzheimer's disease-related neurofibrillary changes. Neurobiol Aging 16: 271-278; discussion 278-284

18. Iqbal K, Grundke-lqbal I (1991) Ubiquitination and abnormal phosphorylation of paired helical filaments in Alzheimer's disease. Mol Neurobiol 5: 399-410

19. Ercan E, Eid S, Weber C, Kowalski A, Bichmann M, Behrendt A, Matthes F, Krauss S, Reinhardt P, Fulle Set al (2017) A validated antibody panel for the characterization of tau post-translational modifications. Mol Neurodegener 12: 87 https://doi.org/10.1186/s13024-017-0229-1

20. Wang Y, Mandelkow E (2016) Tau in physiology and pathology. Nat Rev Neurosci 17: 5-21 https://doi.org/10.1038/nrn.2015.1

21. Martin L, Latypova X, Terro F (2011) Post-translational modifications of tau protein: implications for Alzheimer's disease. Neurochem Int 58: 458-471 https://doi.org/10.1016/..neuint.2010.12.023

22. Despres C, Byrne C, Qi H, Cantrelle FX, Huvent I, Chambraud B, Baulieu EE, Jacquot Y, Landrieu I, Lippens Get al (2017) Identification of the Tau phosphorylation pattern that drives its aggregation. Proc Natl Acad Sci U S A 114: 9080-9085 https://doi.org/10.1073/pnas.1708448114

23. Maeda S, Sahara N, Saito Y, Murayama S, Ikai A, Takashima A (2006) Increased levels of granular tau oligomers: an early sign of brain aging and Alzheimer's disease. Neurosci Res 54: 197-201 https:/doi.org/10.1016/..neures.2005.11.009

24. Patterson KR, Remmers C, Fu Y, Brooker S, Kanaan NM, Vana L, Ward S, Reyes JF, Philibert K, Glucksman MJet al (2011) Characterization of prefibrillar Tau oligomers in vitro and in Alzheimer disease. J Biol Chem 286: 2306323076 https://doi.org/10.1074/jbc.M111.237974

25. Lasagna-Reeves CA, Castillo-Carranza DL, Sengupta U, Sarmiento J, Troncoso J, Jackson GR, Kayed R (2012) Identification of oligomers at early stages of tau aggregation in Alzheimer's disease. FASEB J 26: 1946-1959 https://doi. org/10.1096/fj.11-199851

26. Meraz-Rios MA, Lira-De Leon KI, Campos-Pena V, De Anda-Hernandez MA, Mena-Lopez R (2010) Tau oligomers and aggregation in Alzheimer's disease.
J Neurochem 112: 1353-1367 https://doi.org/10.1111/j.1471-4159.2009. 06511.x

27. Takashima A (2013) Tauopathies and tau oligomers. J Alzheimers Dis 37: 565-568 https://doi.org/10.3233/JAD-130653

28. d'Orange M, Auregan G, Cheramy D, Gaudin-Guerif M, Lieger S, Guillermier M, Stimmer L, Josephine C, Herard AS, Gaillard MCet al (2018) Potentiating tangle formation reduces acute toxicity of soluble tau species in the rat. Brain 141: 535-549 https://doi.org/10.1093/brain/awx342

29. Michel CH, Kumar S, Pinotsi D, Tunnacliffe A, St George-Hyslop P, Mandelkow E, Mandelkow EM, Kaminski CF, Kaminski Schierle GS (2014) Extracellular monomeric tau protein is sufficient to initiate the spread of tau protein pathology. J Biol Chem 289: 956-967 https://doi.org/10.1074/jbc.M113.515445

30. Sharma AM, Thomas TL, Woodard DR, Kashmer OM, Diamond MI (2018) Tau monomer encodes strains. Elife 7: https://doi.org/10.7554/eLife.37813

31. Mirbaha H, Chen D, Morazova OA, Ruff KM, Sharma AM, Liu X, Goodarzi M, Pappu RV, Colby DW, Mirzaei Het al (2018) Inert and seed-competent tau monomers suggest structural origins of aggregation. Elife 7: https://doi.org/ 10.7554/eLife.36584

32. Castillo-Carranza DL, Sengupta U, Guerrero-Munoz MJ, Lasagna-Reeves CA, Gerson JE, Singh G, Estes DM, Barrett AD, Dineley KT, Jackson GRet al (2014) Passive immunization with Tau oligomer monoclonal antibody reverses tauopathy phenotypes without affecting hyperphosphorylated neurofibrillary tangles. J Neurosci 34: 4260-4272 https://doi.org/10.1523/ JNEUROSCI.3192-13.2014

33. Jicha GA, Bowser R, Kazam IG, Davies P (1997) Alz-50 and MC-1, a new monoclonal antibody raised to paired helical filaments, recognize conformational epitopes on recombinant tau. J Neurosci Res 48: 128-132

34. Hyman BT, Van Hoesen GW, Wolozin BL, Davies P, Kromer L, Damasio AR (1988) Alz-50 antibody recognizes Alzheimer-related neuronal changes. Ann Neurol 23: 371-379 https://doi.org/10.1002/ana.410230410

35. Drewes G, Trinczek B, Illenberger S, Biernat J, Schmitt-UIms G, Meyer HE, Mandelkow EM, Mandelkow E (1995) Microtubule-associated protein/ microtubule affinity-regulating kinase (p110mark). A novel protein kinase that regulates tau-microtubule interactions and dynamic instability by phosphorylation at the Alzheimer-specific site serine 262. J Biol Chem 270: 7679-7688

36. Sengupta A, Kabat J, Novak M, Wu Q, Grundke-lqbal I, lqbal K (1998) Phosphorylation of tau at both Thr 231 and Ser 262 is required for maximal inhibition of its binding to microtubules. Arch Biochem Biophys 357: 299309 https://doi.org/10.1006/abbi.1998.0813

37. Liu F, Li B, Tung EJ, Grundke-lqbal I, Iqbal K, Gong CX (2007) Site-specific effects of tau phosphorylation on its microtubule assembly activity and selfaggregation. Eur J Neurosci 26: 3429-3436 https://doi.org/10.1111/j.14609568.2007.05955.x

38. Haase C, Stieler JT, Arendt T, Holzer M (2004) Pseudophosphorylation of tau protein alters its ability for self-aggregation. J Neurochem 88: 1509-1520

39. Guillozet-Bongaarts AL, Cahill ME, Cryns VL, Reynolds MR, Berry RW, Binder LI (2006) Pseudophosphorylation of tau at serine 422 inhibits caspase cleavage: in vitro evidence and implications for tangle formation in vivo. $J$ Neurochem 97: 1005-1014 https://doi.org/10.1111/j.1471-4159.2006.03784.x

40. Song L, Lu SX, Ouyang X, Melchor J, Lee J, Terracina G, Wang X, Hyde L, Hess JF, Parker EMet al (2015) Analysis of tau post-translational modifications in rTg4510 mice, a model of tau pathology. Mol Neurodegener 10: 14 https://doi.org/10.1186/s13024-015-0011-1

41. Huber W, von Heydebreck A, Sueltmann H, Poustka A, Vingron M (2003) Parameter estimation for the calibration and variance stabilization of microarray data. Stat Appl Genet Mol Biol 2: Article3 https://doi.org/10.2202/1544-6115.1008

42. Ritchie ME, Phipson B, Wu D, Hu Y, Law CW, Shi W, Smyth GK (2015) limma powers differential expression analyses for RNA-sequencing and microarray studies. Nucleic Acids Res 43: e47 https://doi.org/10.1093/nar/gkv007

43. Phipson B, Lee S, Majewski IJ, Alexander WS, Smyth GK (2016) Robust Hyperparameter Estimation Protects against Hypervariable Genes and Improves Power to Detect Differential Expression. Ann Appl Stat 10: 946963 https://doi.org/10.1214/16-AOAS920

44. Necas D, Klapetek P (2012) Gwyddion: an open-source software for SPM data analysis. Eur J Phys 10: 181-188

45. Lombardot B https://imagej.net/Interactive_Watershed2019

46. Schindelin J, Arganda-Carreras I, Frise E, Kaynig V, Longair M, Pietzsch T, Preibisch S, Rueden C, Saalfeld S, Schmid Bet al (2012) Fiji: an open-source platform for biological-image analysis. Nat Methods 9: 676-682 https://doi. org/10.1038/nmeth.2019 
47. Schondorf DC, Elschami M, Schieck M, Ercan-Herbst E, Weber C, Riesinger Y, Kalman S, Steinemann D, Ehrnhoefer DE (2019) Generation of an induced pluripotent stem cell cohort suitable to investigate sporadic Alzheimer's Disease. Stem Cell Res 34: 101351 https://doi.org/ 10.1016/j.scr.2018.11.012

48. Shi Y, Kirwan P, Livesey FJ (2012) Directed differentiation of human pluripotent stem cells to cerebral cortex neurons and neural networks. Nat Protoc 7: 1836-1846 https://doi.org/10.1038/nprot.2012.116

49. Courade JP, Angers R, Mairet-Coello G, Pacico N, Tyson K, Lightwood D, Munro R, McMillan D, Griffin R, Baker Tet al (2018) Epitope determines efficacy of therapeutic anti-Tau antibodies in a functional assay with human Alzheimer Tau. Acta Neuropathol 136: 729-745 https://doi.org/10.1007/ s00401-018-1911-2

50. Lasagna-Reeves CA, Castillo-Carranza DL, Sengupta U, Guerrero-Munoz MJ, Kiritoshi T, Neugebauer V, Jackson GR, Kayed R (2012) Alzheimer brainderived tau oligomers propagate pathology from endogenous tau. Sci Rep 2: 700 https://doi.org/10.1038/srep00700

51. Zhang Z, Song M, Liu X, Kang SS, Kwon IS, Duong DM, Seyfried NT, Hu WT, Liu Z, Wang JZet al (2014) Cleavage of tau by asparagine endopeptidase mediates the neurofibrillary pathology in Alzheimer's disease. Nat Med 20: 1254-1262 https://doi.org/10.1038/nm.3700

52. Rowland HA, Hooper NM, Kellett KAB (2018) Modelling Sporadic Alzheimer's Disease Using Induced Pluripotent Stem Cells. Neurochem Res 43: 2179 2198 https://doi.org/10.1007/s11064-018-2663-z

53. Sposito T, Preza E, Mahoney CJ, Seto-Salvia N, Ryan NS, Morris HR, Arber C, Devine MJ, Houlden H, Warner TTet al (2015) Developmental regulation of tau splicing is disrupted in stem cell-derived neurons from frontotemporal dementia patients with the $10+16$ splice-site mutation in MAPT. Hum Mol Genet 24: 5260-5269 https://doi.org/10.1093/hmg/ddv246

54. Frobel J, Hemeda H, Lenz M, Abagnale G, Joussen S, Denecke B, Saric T, Zenke M, Wagner W (2014) Epigenetic rejuvenation of mesenchymal stromal cells derived from induced pluripotent stem cells. Stem Cell Reports 3: 414-422 https://doi.org/10.1016/j.stemcr.2014.07.003

55. Verheyen A, Diels A, Dijkmans J, Oyelami T, Meneghello G, Mertens L, Versweyveld S, Borgers M, Buist A, Peeters Pet al (2015) Using Human iPSCDerived Neurons to Model TAU Aggregation. PLoS One 10: e0146127 https://doi.org/10.1371/journal.pone.0146127

56. Wray S (2017) Modeling tau pathology in human stem cell derived neurons. Brain Pathol 27: 525-529 https://doi.org/10.1111/bpa.12521

57. Alonso A, Zaidi T, Novak M, Grundke-labal I, labal K (2001) Hyperphosphorylation induces self-assembly of tau into tangles of paired helical filaments/straight filaments. Proc Natl Acad Sci U S A 98: 6923-6928 https://doi.org/10.1073/pnas.121119298

58. Tepper K, Biernat J, Kumar S, Wegmann S, Timm T, Hubschmann S, Redecke L, Mandelkow EM, Muller DJ, Mandelkow E (2014) Oligomer formation of tau protein hyperphosphorylated in cells. J Biol Chem 289: 34389-34407 https://doi.org/10.1074/jbc.M114.611368

59. Mair W, Muntel J, Tepper K, Tang S, Biernat J, Seeley WW, Kosik KS, Mandelkow E, Steen H, Steen JA (2016) FLEXITau: Quantifying Posttranslational Modifications of Tau Protein in Vitro and in Human Disease. Anal Chem 88: 3704-3714 https://doi.org/10.1021/acs.analchem.5b04509

60. Augustinack JC, Schneider A, Mandelkow EM, Hyman BT (2002) Specific tau phosphorylation sites correlate with severity of neuronal cytopathology in Alzheimer's disease. Acta Neuropathol 103: 26-35

61. Braak H, Thal DR, Ghebremedhin E, Del Tredici K (2011) Stages of the pathologic process in Alzheimer disease: age categories from 1 to 100 years. J Neuropathol Exp Neurol 70: 960-969 https://doi.org/10.1097/NEN. Ob013e318232a379

62. Neddens J, Temmel M, Flunkert S, Kerschbaumer B, Hoeller C, Loeffler T, Niederkofler V, Daum G, Attems J, Hutter-Paier B (2018) Phosphorylation of different tau sites during progression of Alzheimer's disease. Acta Neuropathol Commun 6: 52 https://doi.org/10.1186/s40478-018-0557-6

63. Hampel H, Buerger K, Zinkowski R, Teipel SJ, Goernitz A, Andreasen N, Sjoegren M, DeBernardis J, Kerkman D, Ishiguro Ket al (2004) Measurement of phosphorylated tau epitopes in the differential diagnosis of Alzheimer disease: a comparative cerebrospinal fluid study. Arch Gen Psychiatry 61: 95-102 https://doi.org/10.1001/archpsyc.61.1.95

64. Sato C, Barthelemy NR, Mawuenyega KG, Patterson BW, Gordon BA, JockelBalsarotti J, Sullivan M, Crisp MJ, Kasten T, Kirmess KMet al (2018) Tau Kinetics in Neurons and the Human Central Nervous System. Neuron 97: 1284-1298 e1287 https://doi.org/10.1016/j.neuron.2018.02.015
65. Collin L, Bohrmann B, Gopfert U, Oroszlan-Szovik K, Ozmen L, Gruninger F (2014) Neuronal uptake of tau/pS422 antibody and reduced progression of tau pathology in a mouse model of Alzheimer's disease. Brain 137: 28342846 https://doi.org/10.1093/brain/awu213

66. Grueninger F, Bohrmann B, Czech C, Ballard TM, Frey JR, Weidensteiner C, von Kienlin M, Ozmen L (2010) Phosphorylation of Tau at S422 is enhanced by Abeta in TauPS2APP triple transgenic mice. Neurobiol Dis 37: 294-306 https://doi.org/10.1016/j.nbd.2009.09.004

67. Wu HY, Kuo PC, Wang YT, Lin HT, Roe AD, Wang BY, Han CL, Hyman BT, Chen YJ, Tai HC (2018) beta-Amyloid Induces Pathology-Related Patterns of Tau Hyperphosphorylation at Synaptic Terminals. J Neuropathol Exp Neurol 77: 814-826 https://doi.org/10.1093/jnen/nly059

68. Khan UA, Liu L, Provenzano FA, Berman DE, Profaci CP, Sloan R, Mayeux R, Duff KE, Small SA (2014) Molecular drivers and cortical spread of lateral entorhinal cortex dysfunction in preclinical Alzheimer's disease. Nat Neurosci 17: 304-311 https://doi.org/10.1038/nn.3606

69. Yang X, Yao C, Tian T, Li X, Yan H, Wu J, Li H, Pei L, Liu D, Tian Qet al (2018) A novel mechanism of memory loss in Alzheimer's disease mice via the degeneration of entorhinal-CA1 synapses. Mol Psychiatry 23: 199-210 https://doi.org/10.1038/mp.2016.151

70. Liguri G, Nassi P, Taddei N, Nediani C, Ramponi G (1988) Post-mortem modifications of the specific activity of some brain enzymes. Neurosci Lett 85: $244-248$

71. Livesey FJ (2014) Human stem cell models of dementia. Hum Mol Genet 23 : R35-39 https://doi.org/10.1093/hmg/ddu302

72. Mertens J, Paquola ACM, Ku M, Hatch E, Bohnke L, Ladjevardi S, McGrath S, Campbell B, Lee H, Herdy JRet al (2015) Directly Reprogrammed Human Neurons Retain Aging-Associated Transcriptomic Signatures and Reveal Age-Related Nucleocytoplasmic Defects. Cell Stem Cell 17: 705-718 https:// doi.org/10.1016/j.stem.2015.09.001

73. Hossini AM, Megges M, Prigione A, Lichtner B, Toliat MR, Wruck W, Schroter F, Nuernberg P, Kroll H, Makrantonaki Eet al (2015) Induced pluripotent stem cell-derived neuronal cells from a sporadic Alzheimer's disease donor as a model for investigating AD-associated gene regulatory networks. BMC Genomics 16: 84 https://doi.org/10.1186/s12864-015-1262-5

74. Israel MA, Yuan SH, Bardy C, Reyna SM, Mu Y, Herrera C, Hefferan MP, Van Gorp S, Nazor KL, Boscolo FSet al (2012) Probing sporadic and familial Alzheimer's disease using induced pluripotent stem cells. Nature 482: 216 220 https://doi.org/10.1038/nature10821

75. Moore S, Evans LD, Andersson T, Portelius E, Smith J, Dias TB, Saurat N, McGlade A, Kirwan P, Blennow Ket al (2015) APP metabolism regulates tau proteostasis in human cerebral cortex neurons. Cell Rep 11: 689-696 https://doi.org/10.1016/j.celrep.2015.03.068

76. Muratore CR, Rice HC, Srikanth $P$, Callahan DG, Shin T, Benjamin LN, Walsh DM, Selkoe DJ, Young-Pearse TL (2014) The familial Alzheimer's disease APPV717I mutation alters APP processing and Tau expression in IPSCderived neurons. Hum Mol Genet 23: 3523-3536 https://doi.org/10.1093/ hmg/ddu064

77. Volpato V, Smith J, Sandor C, Ried JS, Baud A, Handel A, Newey SE, Wessely F, Attar M, Whiteley Eet al (2018) Reproducibility of Molecular Phenotypes after Long-Term Differentiation to Human iPSC-Derived Neurons: A MultiSite Omics Study. Stem Cell Reports 11: 897-911 https://doi.org/10.1016/j. stemcr.2018.08.013

\section{Publisher's Note}

Springer Nature remains neutral with regard to jurisdictional claims in published maps and institutional affiliations.

\section{Ready to submit your research? Choose BMC and benefit from:}

- fast, convenient online submission

- thorough peer review by experienced researchers in your field

- rapid publication on acceptance

- support for research data, including large and complex data types

- gold Open Access which fosters wider collaboration and increased citations

- maximum visibility for your research: over $100 \mathrm{M}$ website views per year

At $\mathrm{BMC}$, research is always in progress.

Learn more biomedcentral.com/submission 